\title{
Searching for continuous gravitational wave sources in binary systems
}

\author{
Sanjeev V. Dhurandhar* \\ Inter-University Centre for Astronomy and Astrophysics, Post Bag 4, Ganeshkhind, Pune 411007, India \\ and Max Planck Institut für Gravitationsphysik, Albert Einstein Institut, Am Mühlenberg 5, D-14476 Golm, Germany \\ Alberto Vecchio ${ }^{\dagger}$ \\ Max Planck Institut für Gravitationsphysik, Albert Einstein Institut, Am Mühlenberg 5, D-14476 Golm, Germany
}

(Received 23 November 2000; published 18 May 2001)

\begin{abstract}
We consider the problem of searching for continuous gravitational wave (cw) sources orbiting a companion object. This issue is of particular interest because the Low mass X-ray binaries (LMXB's), and among them Sco $\mathrm{X}-1$, the brightest $\mathrm{X}$-ray source in the sky, might be marginally detectable with $\approx 2$ y coherent observation time by the Earth-based laser interferometers expected to come on line by 2002 and clearly observable by the second generation of detectors. Moreover, several radio pulsars, which could be deemed to be cw sources, are found to orbit a companion star or planet, and the LIGO-VIRGO-GEO600 network plans to continuously monitor such systems. We estimate the computational costs for a search launched over the additional five parameters describing generic elliptical orbits (up to $e \leq 0.8$ ) using match filtering techniques. These techniques provide the optimal signal-to-noise ratio and also a very clear and transparent theoretical framework. Since matched filtering will be implemented in the final and the most computationally expensive stage of the hierarchical strategies, the theoretical framework provided here can be used to determine the computational costs. In order to disentangle the computational burden involved in the orbital motion of the cw source from the other source parameters (position in the sky and spin down) and reduce the complexity of the analysis, we assume that the source is monochromatic (there is no intrinsic change in its frequency) and its location in the sky is exactly known. The orbital elements, on the other hand, are either assumed to be completely unknown or only partly known. We provide ready-to-use analytical expressions for the number of templates required to carry out the searches in the astrophysically relevant regions of the parameter space and how the computational cost scales with the ranges of the parameters. We also determine the critical accuracy to which a particular parameter must be known, so that no search is needed for it; we provide rigorous statements, based on the geometrical formulation of data analysis, concerning the size of the parameter space so that a particular neutron star is a one-filter target. This result is formulated in a completely general form, independent of the particular kind of source, and can be applied to any class of signals whose waveform can be accurately predicted. We apply our theoretical analysis to Sco X-1 and the 44 neutron stars with binary companions which are listed in the most updated version of the radio pulsar catalog. For up to $\approx 3 \mathrm{~h}$ of coherent integration time, Sco X- 1 will need at most a few templates; for 1 week integration time the number of templates rapidly rises to $\simeq 5$ $\times 10^{6}$. This is due to the rather poor measurements available today of the projected semi-major axis and the orbital phase of the neutron star. If, however, the same search is to be carried out with only a few filters, then more refined measurements of the orbital parameters are called for-an improvement of about three orders of magnitude in the accuracy is required. Further, we show that the five NS's (radio pulsars) for which the upper limits on the signal strength are highest require no more than a few templates each and can be targeted very cheaply in terms of CPU time. Blind searches of the parameter space of orbital elements are, in general, completely un-affordable for present or near future dedicated computational resources, when the coherent integration time is of the order of the orbital period or longer. For wide binary systems, when the observation covers only a fraction of one orbit, the computational burden reduces enormously, and becomes affordable for a significant region of the parameter space.
\end{abstract}

DOI: 10.1103/PhysRevD.63.122001

PACS number(s): 04.80.Nn, 95.55.Ym, 95.75.Pq, 97.60.Gb

\section{INTRODUCTION}

The construction of several large-scale interferometric gravitational wave detectors, with optimal sensitivity in the frequency window $\sim 10 \mathrm{~Hz}$ to $1 \mathrm{kHz}$ is close to completion. For a period extending up to a year, starting from the end of

\footnotetext{
*Electronic address: sdh@iucaa.ernet.in

†Electronic address: vecchio@aei-potsdam.mpg.de
}

the year 2000, engineering data runs will be carried out in order to test and debug the detector components. Finally, between 2002 and 2004 the interferometers will carry out the first set of "science" data runs with the realistic goal of directly observing gravitational waves (GW's). This initial phase will be followed by substantial upgrades on most of the instruments aimed at reaching better sensitivity and larger observational bands. The projects include the Laser Interferometer Gravitational-wave Observatory (LIGO) in the U.S.A. consisting of two facilities, one at Hanford (WA) 
and the other at Livingston (LA), hosting two 4-km and one 2-km interferometers [1]; VIRGO, a French-Italian project located at Cascina, near Pisa, Italy, which consists of a 3-km detector (and currently running about one to two years behind the time frame presented above) [2]; GEO600, a German-British 0.6-km interferometer located at Ruthe, near Hannover, Germany [3]; in Japan, the TAMA project is currently running a medium scale interferometric detector of arm length $300 \mathrm{~m}$, and is planning to extend the base line to $3 \mathrm{~km}$ and carry out other substantial improvements on the instrument within the next few years [4]; finally, the ACIGA consortium will build, if funding is approved, a $500 \mathrm{~m}$ interferometer (AIGO500) near Perth, Australia [5]. In the meantime, a number of existing resonant bar detectors are steadily increasing their sensitivity in narrow bandwidths $(\sim 1 \mathrm{~Hz})$ covering the $\mathrm{kHz}$ spectral window [6] and serious efforts are being made to fly, possibly by 2010, a space-borne laser interferometer [7] (LISA: the Laser Interferometer Space Antenna). LISA will open the low frequency window $10^{-5}$ $10^{-2} \mathrm{~Hz}$, currently accessible only via the Doppler tracking of interplanetary spacecraft [8], and is projected to have sensitivity several orders of magnitude better.

Building and running such powerful machines represents an enormous enterprise, which started over 30 years ago. However, analyzing the large amount of data-several Mbytes per second - and digging out with high confidence astrophysical signals from the noise which severely corrupts the data, presents its own challenges. In fact, over the past few years, interest has been growing in the area of GW data analysis, which is now regarded as one of the key aspects for the successful detection of GW's.

Several types of GW sources have been envisaged which could be directly observed by Earth-based detectors (see [9-12] and references therein for recent reviews): (i) burst sources-such as binary systems of neutron stars (NS's) and/or black holes (BH's) in their in-spiral phase, BH-BH and/or BH-NS mergers-and supernova explosions-whose signals last for a time much shorter, typically between a few milli-seconds and a few minutes, than the planned observational time; (ii) stochastic backgrounds of radiation, either of primordial or astrophysical origin, and (iii) continuous wave (cw) sources-e.g. rapidly rotating neutron stars-where a weak deterministic signal is continuously present in the data stream.

In this paper we deal with data analysis issues regarding $\mathrm{cw}$ sources. More specifically, we investigate the computational load involved in filtering the data stream to search for monochromatic radiation emitted by a neutron star orbiting a companion object.

\section{A. Data analysis challenge}

Continuous wave emitters pose one of the most computationally intensive problems in GW data analysis. The weakness of the expected signal requires very long observation times, of the order of 1 year (or possibly more) for accumulating enough signal-to-noise ratio (SNR) so that detection is possible. During the observation time, a monochromatic signal in the source reference frame is Doppler modulated by the motion of the detector carried by the spinning Earth orbiting the Sun. The emitted energy is spread over $\simeq 2$ $\times 10^{6}\left(T / 10^{7} \mathrm{sec}\right)^{2}(f / 1 \mathrm{kHz})$ frequency bins of width $\Delta f$ $=1 / T$, where $T$ is the time of observation (the formula holds for $T$ up to 6 months; after that the number of bins increases linearly with $T$ ). In order to recover the whole power in one frequency bin one has to "correct" the recorded data stream for each possible source position in the sky. The problem is made worse if the intrinsic frequency of the source changes, say due to spindown. Then the power is spread over 3 $\times 10^{6}\left(\tau / 10^{3} \mathrm{yr}\right)^{-1}\left(T / 10^{7} \mathrm{sec}\right)^{2}(f / 1 \mathrm{kHz})$ bins, where $\tau$ $=f / \dot{f}$ is (twice) the spin-down age of the NS. Indeed, one then needs to correct, in addition, for this effect searching through one or more spin-down parameters. It is clear that searches for cw's are limited by the available computational resources $[13,14]$. The optimal technique of tracking coherently the signal phase-matched filtering - is not affordable for integration times longer than $\sim 1-10$ days (the integration time depends on the range of parameters over which the search is being carried out). So far, the only viable strategy for a "blind" search over a wide range of parameters is based on a hierarchical structure $[15,16]$. In this type of search, coherent and incoherent search stages are alternated in order to identify candidate signals - and the relevant parameter range- " cheaply" by a suboptimal algorithm. Then, the candidate signals are followed up with a coherent and computationally expensive search over the entire observation time-this step constitutes the bottle neck for processing power-to recover the optimal SNR.

However, because of the large computational burden, the algorithms investigated so far have been restricted to isolated NS's. The problem of searching for a cw emitter orbiting a companion object is simply considered computationally intractable, as it would require up to five more search parameters which would compound the already enormous computational cost.

To gain insight into the additional Doppler modulation introduced on the signal phase by the source motion around a companion, let us consider a NS, of mass $m_{\mathrm{NS}}=1.4 M_{\odot}$, with an orbiting companion object of mass $m_{2}$ and period $P$. During the observation time $T$, the NS velocity changes by $\Delta v=(2 \pi / P)^{4 / 3}\left(G m_{\mathrm{NS}}\right)^{1 / 3} q /(1+q)^{2 / 3} T$, where $q$ $\equiv m_{2} / m_{\mathrm{NS}}$. The frequency is Doppler shifted by $\Delta_{D} f$ $= \pm f \Delta v / c$ which has to be compared to the frequency resolution $\Delta f$. (Here and after, $G$ denotes the Newton's gravitational constant and $c$ denotes the speed of light or actually GW.) The maximum observational time $T_{\max }$ after which one needs to correct for the cw's source orbital motion is, therefore,

$$
\begin{aligned}
T_{\max } \simeq & 131.6\left[\frac{(1+q)^{1 / 3}}{\sqrt{q}}\right]\left(\frac{P}{1 \text { day }}\right)^{2 / 3}\left(\frac{m_{N S}}{1.4 M_{\odot}}\right)^{-1 / 6} \\
& \times\left(\frac{f}{1 \mathrm{kHz}}\right)^{-1 / 2} \mathrm{sec} .
\end{aligned}
$$

Of course, this time scale can vary by orders of magnitude, depending on the NS companion and the orbital period. For the relevant astrophysical situations we have 


$$
T_{\max } \simeq \begin{cases}1.2 \times 10^{5}\left(\frac{P}{10^{7} \mathrm{sec}}\right)^{2 / 3}\left(\frac{m_{2}}{10^{-3} M_{\odot}}\right)^{-1 / 2}\left(\frac{f}{1 \mathrm{kHz}}\right)^{-1 / 2} \mathrm{sec} & \left(m_{2} \ll m_{\mathrm{NS}}\right), \\ 1.0 \times 10^{2}\left(\frac{P}{12 \mathrm{~h}}\right)^{2 / 3}\left(\frac{f}{1 \mathrm{kHz}}\right)^{-1 / 2} \mathrm{sec} & \left(m_{2} \simeq m_{\mathrm{NS}}\right), \\ 4.4 \times 10^{2}\left(\frac{P}{10 \text { days }}\right)^{2 / 3}\left(\frac{m_{2}}{10 M_{\odot}}\right)^{-1 / 6}\left(\frac{f}{1 \mathrm{kHz}}\right)^{-1 / 2} \mathrm{sec} & \left(m_{2} \gg m_{\mathrm{NS}}\right) .\end{cases}
$$

For comparison, we note that the maximum integration time before which the motion of the detector from Earth's rotation about its axis produces a Doppler shift in frequency greater than the frequency resolution bin is $T_{\max }^{(\mathrm{det})} \simeq 50(\mathrm{f} / 1 \mathrm{kHz})^{-1 / 2}$ min. It is then evident that in the case of a cw source orbiting a companion object, the frequency shift is typically more severe and the computational challenge that one faces when searching for the additional 5-dimensional parameter vector describing the binary orbit is considerable.

\section{B. Expected sources and motivation}

The characteristic amplitude of the continuous signal emitted by a triaxial NS, rotating about a principal axis with period $P_{r}$ and frequency $f_{r}=1 / P_{r}$, reads [9]

$$
h_{\mathrm{c}} \simeq 7.7 \times 10^{-26}\left(\frac{\epsilon_{e}}{10^{-6}}\right)\left(\frac{D}{10 \mathrm{kpc}}\right)^{-1}\left(\frac{f_{r}}{500 \mathrm{~Hz}}\right)^{2},
$$

where $\epsilon_{e}$ is the equatorial ellipticity of the star, and we have taken the standard value $10^{45} \mathrm{~g} \mathrm{~cm}^{2}$ for the NS moment of inertia. In Eq. (1.3) we assume that the GW energy is released at exactly twice the rotation frequency, so that $f$ $=2 f_{r}$.

The characteristic amplitude has to be compared with the noise fluctuations,

$$
h_{\mathrm{n}}=\sqrt{\frac{S_{n}(f)}{T}}=10^{-25}\left(\frac{S_{n}(f)}{10^{-43} \mathrm{~Hz}^{-1}}\right)^{1 / 2}\left(\frac{T}{10^{7} \mathrm{sec}}\right)^{-1 / 2},
$$

in a resolution bin, where $S_{n}(f)$ is the noise spectral density of the instrument. A signal is detected if $h_{\mathrm{c}} \gtrless k h_{\mathrm{n}}$, where the multiplicative factor $k$, of order of a few, depends on the number of filters used in the analysis and on the (subjective) confidence level of the measurement. The strength of the signal depends crucially on the $\epsilon_{e}$, which is a measure of the non-axis symmetry of a star. Although large uncertainties exist in the maximum value that $\epsilon_{e}$ can achieve, theoretical studies of the braking strain of NS crusts suggest that $\epsilon_{e}$ $\lesssim 10^{-5}$. One can observationally constrain $\epsilon_{e}$ if the NS rotational period $P_{r}$ and its first time derivative $\dot{P}_{r}$ are known:

$$
\epsilon_{e} \leqslant 5.1 \times 10^{-7}\left(\frac{P_{r}}{2 \mathrm{msec}}\right)^{3 / 2}\left(\frac{P_{r}}{10^{-15}}\right)^{1 / 2} .
$$

This yields an upper limit on the amplitude of the emitted GW's, by assuming that all energy is lost through gravitational wave emission. By combining Eqs. (1.3) and (1.5), we can immediately place the upper limit

$$
h_{\mathrm{c}} \leqslant 3.9 \times 10^{-26}\left(\frac{f_{r}}{500 \mathrm{~Hz}}\right)^{1 / 2}\left(\frac{\dot{P}_{r}}{10^{-15}}\right)^{1 / 2}\left(\frac{D}{10 \mathrm{kpc}}\right)^{-1} .
$$

The comparison of $h_{\mathrm{c}}$ for some known NS's with $h_{\mathrm{n}}$ of the laser interferometers is shown in Fig. 1.

In Table I we also show some of the parameters of the binary radio pulsars which are known so far. It is interesting to notice that a bunch of systems are within the reach of the first generation of instruments for coherent observation times from 15 months to $4 \mathrm{yr}$, and well within the enhanced con-

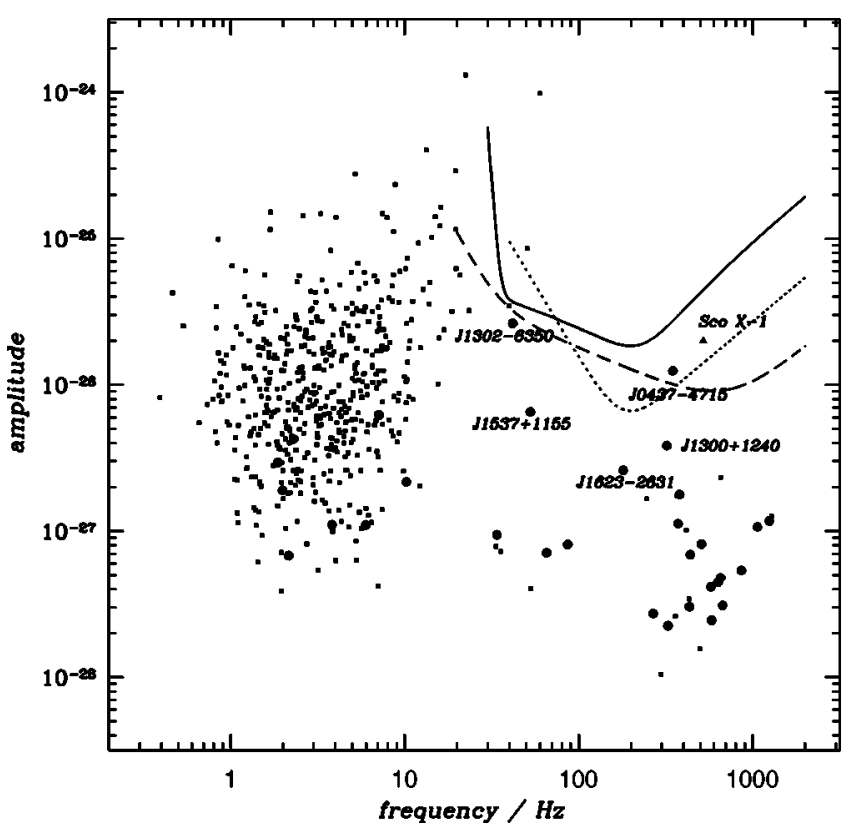

FIG. 1. Upper limits on the GW amplitude from known radio pulsars and Sco X-1. The plot shows the upper limit on $h_{\mathrm{c}}$, Eq. (1.6), for the neutron stars included in the latest version of the published radio pulsar catalogue [17], for which the spin down and the distance are known (isolated pulsars, squares; binary pulsars, bold bullets), and the estimated value of $h_{\mathrm{c}}$ for Sco X-1 (triangle), Eq. (1.7). We also show the noise amplitude $h_{\mathrm{n}}$ for an observation time $T=10^{7} \mathrm{sec}$, for the first generation detectors (GEO600, solid line; LIGO I, dotted line; VIRGO, dashed line). 
TABLE I. Neutron stars detected as radio pulsars in binary systems. The table shows the main parameters of the 44 known neutron stars detected as radio pulsars orbiting a companion object listed in the catalog of Taylor et al. [17]. We list the gravitational wave frequency $f$-assumed to be exactly twice the radio frequency-its first time derivative $\dot{f}$, the estimated distance $D$ from the solar system and the spin-down age (we do not give all significant digits). The orbital parameters-the orbital period $P$, the projection of the semi-major axis along the line of sight $a_{\mathrm{p}}$ and the eccentricity $e-$ are tabulated with their respective errors (in parentheses). Notice that out of the 44 known systems, seven sources radiate at a frequency $<10 \mathrm{~Hz}$ and therefore are not observable by the (so far) planned ground-based experiments.

\begin{tabular}{|c|c|c|c|c|c|c|c|c|}
\hline PSR J & PSR B & $\begin{array}{c}f \\
(\mathrm{~Hz})\end{array}$ & $\begin{array}{c}\dot{f} \\
\left(10^{-15} \sec ^{-2}\right)\end{array}$ & $\begin{array}{c}D \\
(\mathrm{kpc})\end{array}$ & $\log ($ age $/ y r)$ & $\begin{array}{c}P \\
\text { (days) }\end{array}$ & $\begin{array}{c}a_{\mathrm{p}} \\
(\mathrm{sec})\end{array}$ & $e$ \\
\hline 0024-7204E & $0021-72 \mathrm{E}$ & 565.6 & - & 4.5 & - & $2.256844(3)$ & $1.978(3)$ & $0.000(3)$ \\
\hline 0024-7204I & $0021-72 I$ & 573.9 & - & 4.5 & - & $0.226(3)$ & $0.039(2)$ & $0.00(5)$ \\
\hline 0024-7204J & $0021-72 J$ & 952.1 & - & 4.5 & - & $0.120665(2)$ & $0.0402(2)$ & $0.00(5)$ \\
\hline 0034-0534 & & 1065.4 & 3.8 & 1.0 & 9.6 & $1.58928180(3)$ & $1.437768(5)$ & $0.0000(1)$ \\
\hline 0045-7319 & & 2.2 & 10.5 & 57.0 & 6.5 & $51.169226(3)$ & $174.2540(8)$ & $0.807995(5)$ \\
\hline $0218+4232$ & & 860.9 & 27.8 & 5.8 & 8.7 & $2.02885(1)$ & $1.9844(1)$ & $0.00000(2)$ \\
\hline $0437-4715$ & & 347.4 & 3.4 & 0.1 & 9.2 & $5.741042329(12)$ & $3.3666787(14)$ & $0.0000187(10)$ \\
\hline 0613-0200 & & 653.2 & 2.3 & 2.2 & 9.6 & $1.19851251(2)$ & $1.09145(2)$ & $0.000000(22)$ \\
\hline $0700+6418$ & $0655+64$ & 10.2 & 0.04 & 0.5 & 9.7 & 1.028669703 & $4.125612(5)$ & $0.0000075(11)$ \\
\hline $0751+1807$ & & 574.9 & 1.3 & 2.0 & 9.8 & 0.2631442679 & $0.396615(2)$ & $0.0000(1)$ \\
\hline $0823+0159$ & $0820+02$ & 2.3 & 0.3 & 1.4 & 8.1 & $1232.47(12)$ & $162.1466(7)$ & $0.011868(7)$ \\
\hline $1012+5307$ & & 380.5 & 1.1 & 0.5 & 9.8 & 0.604672713 & $0.581816(6)$ & $0.00000(2)$ \\
\hline $1022+10$ & & 121.6 & - & 0.6 & - & $7.80513014(8)$ & $16.765412(6)$ & $0.0000977(6)$ \\
\hline $1045-4509$ & & 267.6 & 0.7 & 3.2 & 9.8 & $4.0835291(1)$ & $3.015107(9)$ & $0.000019(6)$ \\
\hline $1300+1240$ & $1257+12$ & 321.6 & 5.9 & 0.6 & 8.9 & $66.536(1)$ & $0.0013106(6)$ & $0.0182(9)$ \\
\hline $1302-6350$ & $1259-63$ & 41.9 & 1994.5 & 4.6 & 5.5 & $1236.72359(5)$ & $1296.580(2)$ & $0.869931(1)$ \\
\hline $1312+1810$ & $1310+18$ & 60.3 & - & 18.9 & - & $255.8(6)$ & $84.2(7)$ & $0.002(3)$ \\
\hline $1455-3330$ & & 250.4 & - & 0.7 & - & $76.17458(2)$ & $32.36222(6)$ & $0.000167(2)$ \\
\hline $1518+4904$ & & 48.9 & - & 0.7 & - & $8.6340047(8)$ & $20.043982(8)$ & $0.2494848(7)$ \\
\hline $1537+1155$ & $1534+12$ & 52.8 & 3.4 & 0.7 & 8.4 & 0.4207372998 & $3.729468(9)$ & $0.2736779(6)$ \\
\hline $1623-2631$ & $1620-26$ & 180.6 & 12.9 & 1.8 & 8.3 & $191.442819(2)$ & $64.809476(13)$ & $0.0253147(5)$ \\
\hline $1640+2224$ & & 632.2 & 0.6 & 1.2 & 10.2 & $175.460668(2)$ & $55.329722(2)$ & $0.00079742(5)$ \\
\hline $1641+3627 B$ & $1639+36 B$ & 566.9 & - & 7.7 & - & $1.259113(3)$ & $1.389(2)$ & $0.005(5)$ \\
\hline $1643-1224$ & & 432.7 & 3.1 & 4.9 & 9.3 & $147.01743(6)$ & $25.07260(4)$ & $0.000506(2)$ \\
\hline $1713+0747$ & & 437.6 & 0.8 & 1.1 & 9.9 & $67.82512988(2)$ & 32.342413 (1) & $0.00007492(2)$ \\
\hline $1721-1936$ & $1718-19$ & 2.0 & 3.2 & 11.6 & 7.0 & 0.2582735 & $0.3526(8)$ & $0.000(5)$ \\
\hline $1748-2446 \mathrm{~A}$ & $1744-24 \mathrm{~A}$ & 173.0 & -0.3 & 7.1 & - & 0.0756461170 & 0.11963 (2) & $0.0000(12)$ \\
\hline $1803-2712$ & $1800-27$ & 6.0 & 0.3 & 3.6 & 8.5 & $406.781(2)$ & $58.9397(18)$ & $0.000507(6)$ \\
\hline 1804-0735 & $1802-07$ & 86.6 & 1.8 & 3.2 & 8.9 & $2.6167634(5)$ & 3.92047 (4) & $0.211999(15)$ \\
\hline $1804-2718$ & & 214.1 & - & 1.2 & - & $11.15(1)$ & $7.20(4)$ & $0.00(1)$ \\
\hline $1823-1115$ & $1820-11$ & 7.1 & 35.2 & 6.3 & 6.5 & $357.7622(3)$ & $200.672(3)$ & $0.79462(1)$ \\
\hline 1834-0010 & $1831-00$ & 3.8 & 0.1 & 2.6 & 8.8 & $1.811103(2)$ & $0.7231(8)$ & $0.000(4)$ \\
\hline $1857+0943$ & $1855+09$ & 373.0 & 1.2 & 0.9 & 9.7 & $12.3271711905(6)$ & 9.2307802 (4) & $0.00002168(5)$ \\
\hline $1910+0004$ & & 552.7 & - & 4.1 & - & $0.140996(1)$ & $0.038(2)$ & $0.00(1)$ \\
\hline $1915+1606$ & $1913+16$ & 33.9 & 4.9 & 7.1 & 8.0 & $0.322997462736(7)$ & $2.3417592(19)$ & 0.6171308 \\
\hline $1955+2908$ & $1953+29$ & 326.1 & 1.6 & 5.4 & 9.5 & $117.349097(3)$ & $31.412686(5)$ & 0.0003304 \\
\hline $1959+2048$ & $1957+20$ & 1244.2 & 13.0 & 1.5 & 9.2 & $0.3819666389(13)$ & $0.892268(8)$ & $0.00000(4)$ \\
\hline $2019+2425$ & & 508.3 & 0.9 & 0.9 & 9.9 & $76.5116347(1)$ & $38.767625(1)$ & $0.00011111(6)$ \\
\hline $2033+17$ & & 336.2 & - & 1.4 & - & $56.2(1)$ & $20.07(8)$ & $0.00(5)$ \\
\hline $2130+1210 \mathrm{C}$ & $2127+11 C$ & 65.5 & 10.7 & 10.0 & 8.0 & $0.335282052(6)$ & $2.520(3)$ & $0.68141(2)$ \\
\hline $2145-0750$ & & 124.6 & - & 0.5 & - & $6.83890256(8)$ & $10.16411(1)$ & $0.000021(2)$ \\
\hline $2229+2643$ & & 671.6 & 0.4 & 1.4 & 10.4 & $93.015895(2)$ & $18.912519(5)$ & $0.0002550(4)$ \\
\hline $2305+4707$ & $2303+46$ & 1.9 & 1.0 & 4.4 & 7.5 & 12.33954454 (17) & $32.6878(3)$ & $0.658369(9)$ \\
\hline $2317+1439$ & & 580.5 & 0.4 & 1.9 & 10.4 & $2.459331464(2)$ & $2.3139483(9)$ & $0.0000005(7)$ \\
\hline
\end{tabular}


figuration sensitivity. It is also worth noticing that they represent a fair sample of orbital companions, as they include NS-NS binaries, NS-white-dwarf binaries, NS-mainsequence stars and NS-planet. However, the actual signal strain could be orders of magnitude below the upper limit (1.6).

A far more promising scenario is the accretion of hot material onto the NS surface. Here the induced quadrupole moment is directly related to the accretion rate, which can be copious. The gravitational energy reservoir, moreover, can be continuously replenished, if persistent accretion occurs. The key idea behind this scenario is that gravitational wave radiation can balance the torque due to accretion, and was proposed over 20 years ago $[18,19]$. However, it has attracted considerable new interest in the past two years and has been fully revitalized by the launch of the Rossi X-ray Timing Explorer, designed for precision timing of accreting NS's.

Observational evidence that low mass $\mathrm{x}$-ray binaries (LMXB's) - binary systems where a compact object accretes material from a low mass companion-in our Galaxy are clustered around a rotation frequency $\approx 300 \mathrm{~Hz}$ led Bildsten [20] to propose a mechanism to explain this behavior. The fundamental idea is that continuous emission of GW's radiates away the angular momentum that is transferred to the NS by the infalling material. The fact that the rate of angular momentum loss through GW's scales as $f^{5}$ provides a very natural justification of the clustering of rotation frequency of several sources. The physical process responsible for producing a net quadrupole moment is the change of composition in the NS crust, which in turn is produced by the temperature gradient caused by the in-falling hot material. Recently, Ushomirsky et al. [21] have posed this initial idea on more solid theoretical grounds. If such a mechanism does operate, LMXB's are extremely interesting candidate sources for Earth-based detectors. Several systems would be detectable by LIGO operating in the "enhanced" configuration (LIGO II), if the detector sensitivity is tuned, through narrow banding, around the emission frequency. In particular, Sco X-1, the most luminous $\mathrm{x}$-ray source in the sky, is possibly marginally detectable by “initial” ' LIGO and GEO600 (the latter in narrow-band configuration), where an integration time of approximately 2 y would be required. The characteristic amplitude for this class of sources is

$$
\begin{aligned}
h_{\mathrm{c}} \simeq & 4 \times 10^{-27}\left(\frac{R_{N S}}{10^{6} \mathrm{~cm}}\right)^{3 / 4}\left(\frac{m_{N S}}{1.4 M_{\odot}}\right)^{-1 / 4} \\
& \times\left(\frac{F_{X}}{10^{-8} \mathrm{erg} \mathrm{cm}^{-2} \mathrm{sec}^{-1}}\right)^{-1 / 2},
\end{aligned}
$$

where $R_{\mathrm{NS}}$ is the typical radius of a neutron star and $F_{X}$ is the $\mathrm{x}$-ray flux.

Data analysis strategies to search for NS's in binary systems might therefore be vital for ground-based detectors. They would supplement the already existing strategies for the several sources mentioned above.

\section{Organization of the paper}

The paper is organized as follows: In Sec. II we set up the parametrized model of the signal based on the two body problem. We then identify the search parameters of the signal, which are essentially the binary orbital elements, for the general case of elliptic orbits, as well as for circular orbits. In Sec. III we discuss the geometrical approach to the data analysis; in Sec. III A we review the method of determining the number of templates which involves the computation of the metric on the signal manifold, and we also state useful properties of the metric which are then used in the later sections. In Sec. III B we present a rigorous criterion, naturally suggested by the geometrical picture, for estimating the number of filters required in targeted searches and to determine whether any particular source is a one-filter target.

Sections IV and V contain the key results of the paper. In Sec. IV we obtain analytic approximate formulas for the number density of filters per unit coordinate volume in the parameter space in the two important regimes of observation time: (i) observations lasting for several orbital periods and (ii) observations lasting a fraction of an orbit. These expressions provide for us the scaling: that is, how the computational cost scales with different parameters. They can also be readily applied to estimate the computational cost of detecting GW's from some observed systems. Further, we also compare these results with the fully numerical computation of the metric determinant (which shows excellent agreement over a wide range of the parameters), and determine the range of applicability of these approximate expressions as a function of the relevant parameters (in particular the time of observation as compared to the orbital period). In the Sec. V we apply the above analysis to known NS's in binary systems, specifically radio pulsars and LMXB's. Given the current (or near future) computational resources, we then determine the systems that could be searched by matched filtering methods. Section VI contains our final remarks and future directions of work.

\section{MODEL}

\section{A. Description of the problem}

In this paper we employ the matched filtering technique for coherent integration of the signal. It provides for us the optimal signal-to-noise ratio that can be achieved with linear data analysis techniques and also an elegant theoretical framework can be given in which the computational costs can be derived in a very transparent way. We have already remarked that the processing power of present or near future computers falls woefully short for carrying out full matchedfilter based searches of cw sources. However, it is important to note that matched filters are implemented in the final stage of every hierarchical strategy that has been devised so far $[15,16]$. Our analysis can be therefore applied to the typically reduced parameter range which is relevant in this final step. Since the final stage accounts for most of the processing power required in the entire search, our results essentially reflect the computational costs involved in the search. 
The amplitude of the GW's is expected to be small for cW sources. Therefore long observation times are imminent for extracting the signal out of the detector noise with a reasonable SNR. The observation times could last for a few months, up to a year or more. During this period of observation it becomes essential to take into account the changes in frequency which result from the variation in relative positions of the source and the detector-Doppler effects-and also those that are intrinsic to the source, such as the spin down. The general idea is (i) to correct for these changes in the phase, (ii) use the fast Fourier transform (FFT) algorithm to compute efficiently the power spectrum and then (iii) look for a statistically significant peak in the power spectrum itself.

The aim of this paper is to estimate the extra computational costs associated with the search for sources in binary systems. In order to disentangle the phase Doppler modulation produced by the source motion around the orbital companion, and limit the complexity of our analysis, we will make the following assumptions:

(i) The GW source radiates perfectly monochromatic GW's at the intrinsic frequency $f_{0}$, that is the frequency in its own rest frame is constant: we ignore the effect of spin-down parameters.

(ii) The location of the source in the sky is perfectly known: the Doppler effect produced by the motion of the Earth spinning on its own axis, orbiting the Sun etc. can be computed and subtracted from the data stream with very little CPU load.

These hypotheses leave us only to grapple with the problem of the motion of the source. Notice that they do not affect the generality of our analysis. This approach returns the extra cost involved in searching for NS's in binary systems, for each template of the isolated NS search (with the appropriate number of spin-down parameters and range of parameter values). Our analysis thus provides a solid upper limit on the total computational costs (it ignores possible correlations between the parameters). In fact, if we call $\mathcal{N}_{\text {is }}$ the number of templates to search for an isolated NS (the search parameters are the location in the sky and spin-down coefficients) and $\mathcal{N}$ the number of templates required to search for a perfectly monochromatic source in binary orbit whose location in the sky is assumed to be known, the search parameters being only the orbital elements of the binary system, then the total number of filters $\mathcal{N}_{\mathrm{T}}$ required for a generic source, is bound by the following inequality:

$$
\mathcal{N}_{\mathrm{T}} \leqslant \mathcal{N}_{\text {is }} \times \mathcal{N} .
$$

We take the orbit of the source to be Keplerian, elliptical in shape, but we assume that we do not exactly know, or do not know at all, the orbital parameters. It is possible that a particular parameter or a set of parameters is known so accurately that no search is required over these parameters. Such a situation arises for some of the known radio binary pulsars listed in the published catalog [17]. We then have a targeted search, where one must now search only over rest of the parameters. In the extreme case, all parameters may be known so accurately that only a single Doppler correction needs to be applied (one filter in the language of data analysis). Usually for known systems some orbital elements are known within given error bars. For example, one might know the period of the orbit within certain limits; then these limits provide for us the ranges of the parameters, over which the search must be carried out. On the other hand, we might not know anything at all about the binary system, in which case one must launch a more extensive search with a large number of templates covering the entire region of the parameter space - this is the so-called "blind" search.

\section{B. Two body problem, orbital elements and search parameters}

The essential problem of celestial mechanics is the twobody problem, where one solves for the motion of two point particles of masses $m_{1}$ and $m_{2}$, and position vectors $\mathbf{r}_{1}$ and $\mathbf{r}_{2}$, attracted by their mutual gravitational force. The solution is simple in their center-of-mass frame in terms of the relative position vector $\mathbf{r}=\mathbf{r}_{1}-\mathbf{r}_{2}$. The vector $\mathbf{r}$ lies in a plane and traces out an ellipse; the individual masses trace out similar ellipses scaled by factors depending on the masses:

$$
\mathbf{r}_{1}=\frac{m_{2}}{M} \mathbf{r}, \quad \mathbf{r}_{2}=-\frac{m_{1}}{M} \mathbf{r},
$$

where $M=m_{1}+m_{2}$. Here, we (arbitrarily) take $\mathbf{r}_{1} \equiv \mathbf{r}_{\mathrm{NS}}$ to describe the position of the $\mathrm{cw}$ source we want to detect. We assume that the center of mass of the binary system can be considered at rest, or in uniform motion, with respect to the Solar System barycenter during the period of observation; therefore knowing $\mathbf{r}_{1}$ as a function of time is sufficient for us to compute the GW Doppler phase shift $\phi_{D}(t)$.

It is convenient to set up a Cartesian coordinate system $(\xi, \eta, \zeta)$ attached to the binary source, so that (i) $\mathbf{r}_{1}$ lies in the $(\xi, \eta)$ plane with the origin at the center of the ellipse, (ii) the semi-major axis of the ellipse coincides with the $\xi$ axis, and (iii) the $\zeta$ axis is perpendicular to the orbital plane and points in the direction of the orbital angular momentum. We specify the direction to the detector, in the $(\xi, \eta, \zeta)$ frame, by the unit vector

$$
\hat{\mathbf{n}}=(\sin \epsilon \cos \psi) \hat{\boldsymbol{\xi}}+(\sin \epsilon \sin \psi) \hat{\boldsymbol{\eta}}+(\cos \epsilon) \hat{\boldsymbol{\zeta}},
$$

where $\epsilon$ and $\psi$ are the usual polar angles.

The Doppler phase shift at the detector output therefore reads

$$
\phi_{D}(t)=-\frac{2 \pi f_{0}}{c}\left[\hat{\mathbf{n}} \cdot \mathbf{r}_{1}(t)\right]
$$

where $c \simeq 2.9979 \times 10^{10} \mathrm{~cm} / \mathrm{sec}$ is the speed of light.

The orbit in the $(\xi, \eta)$ plane is given as follows: Let $a$ be the semi-major axis of the elliptical orbit and $e$ the eccentricity; then, the orbit is described by the equations

$$
\begin{aligned}
\xi(t) & =a \cos E(t), \\
\eta(t) & =a \sqrt{1-e^{2}} \sin E(t),
\end{aligned}
$$


where $E$ is the so-called eccentric anomaly, and is a function of the time $t$. It is related to the mean angular velocity $\omega$ and the mean anomaly $M(t)$ by the Kepler equation,

$$
E(t)-e \sin E(t)=\omega t+\alpha \equiv M(t),
$$

where $\alpha$ is an initial phase, $0 \leqslant \alpha<2 \pi$. When $\omega t+\alpha=0$ we have $E=0$ and the mass is closest to the focus $\xi=a e, \eta$ $=0$. These equations describe the orbit in the $(\xi, \eta)$ plane as a function of time, which is determined by the four orbital elements $a, \omega, \alpha, e$. However, the orbit in space requires two additional parameters, namely, the angles $\epsilon$ and $\psi$ mentioned before. Thus, in all we have six orbital elements which specify the orbit in space.

The Doppler phase correction, Eq. (2.4), is obtained from Eq. (2.5), and reads as follows:

$$
\begin{aligned}
\phi_{D}(t)= & -\frac{2 \pi f_{0} a \sin \epsilon}{c}[\cos \psi \cos E(t) \\
& \left.+\sin \psi \sqrt{1-e^{2}} \sin E(t)\right] .
\end{aligned}
$$

The total phase $\Phi$ at the barycenter (we have assumed that the corrections have been made for the Earth's motion) is, therefore,

$$
\Phi(t)=2 \pi f_{0} t+\phi_{D}(t) .
$$

Since we have assumed that we have corrected for the Earth's motion, the $t$ can be regarded as the barycentric time.

For computing the metric in the next section we will require the time averages of the derivatives of the phase with respect to the search parameters. Since the Kepler equation connects $E$ to the time $t$, or $M$ implicitly, $\cos E$ and $\sin E$ are usually expressed as power series in the eccentricity parameter $e$ with harmonics in $M$. Also the time averages are more conveniently computed using $M$. We thus write, formally,

$$
\begin{array}{r}
\cos E=\sum_{k=0}^{\infty} \mathcal{C}_{k}(e) \cos (k M), \\
\sqrt{1-e^{2}} \sin E=\sum_{k=1}^{\infty} \mathcal{S}_{k}(e) \sin (k M),
\end{array}
$$

where $\mathcal{C}_{k}(e)$ and $\mathcal{S}_{k}(e)$ are power series in $e$. In this work we consider expansions up to the 7th power in $e$, which automatically means that we consider 7 harmonics in $M$. Approximation to this order provides fairly accurate results for $e$ up to $\sim 0.8$ (see Sec. IV C on elliptical orbits) which should include most binary systems. Moreover, all binary radio pulsars detected so far have $e<0.8$; cf. Table I. The $\mathcal{C}_{k}(e)$ and $\mathcal{S}_{k}(e)$ are given in Appendix A up to this order.

The parameters over which one must launch a search are not exactly the orbital elements, and need not be of the same number. It is the Doppler phase correction that is observed and so the information about the system that we can glean depends on the combination of the orbital elements that enter into it. The $a$ and $\epsilon$ combine into a single parameter $a \sin \epsilon$ $\equiv a_{\mathrm{p}}$, the projected semi-major axis along the line of sight, which is actually the quantity inferred from astronomical ob- servations. The other search parameters are the remaining orbital elements $\omega, \alpha, e$ and $\psi$. So in the general case, when we do not know any of the parameters exactly, we have a 5-dimensional parameter space to search.

We note that $f_{0}$ is not a search parameter because of the special search technique that is employed [14]: it involves the "stretching" of the time coordinate in such a way, so as to make the signal appear monochromatic in this time coordinate. One then simply takes the FFT to compute the power spectrum, which now is concentrated in a single frequency bin.

The gravitational waveform that we are dealing with, reads (in the barycentric frame)

$$
h(t, \boldsymbol{\Lambda})=\Re\{\mathcal{A} \exp [-i \Phi(t ; \boldsymbol{\Lambda})+i \Psi]\}
$$

where $\Phi(t ; \boldsymbol{\Lambda})$ is given by Eq. (2.8), $\boldsymbol{\lambda}=\left(a_{p}, \omega, \alpha, e, \psi\right)$ is the 5-dimensional vector that refers to the parameters that are required in the discrete mesh search, and $\boldsymbol{\Lambda}=\left(f_{0}, \boldsymbol{\lambda}\right)$. The polarization amplitude $\mathcal{A}$ and the polarization phase $\Psi$ are slowly varying time-dependent functions over the time scale of 1 day and depend on the relative orientations of the source and the detector. In agreement with all the investigations carried out so far, we assume them constant in our analysis. It is expected that these factors can be easily included in the full analysis and will not significantly affect the computational burden [14].

\section{DATA ANALYSIS}

In the geometrical picture [23,24], the signal is a vector in the vector space of data trains and the $\mathrm{N}$-parameter family of signals traces out an $\mathrm{N}$-dimensional manifold which is termed as the signal manifold. The parameters themselves are coordinates on this manifold. One can introduce a metric $\gamma_{j k}$ on the signal manifold which is related to the fractional loss in the SNR when there is a mismatch of parameters between the signal and the filter. The spacing of the grid of filters is decided by the fractional loss due to the imperfect match that can be tolerated. Given the parameter space that one needs to scan, it is then easy to estimate the total number of filters required to carry out the search for the signal. In Sec. III A we first briefly review the method introduced by Brady et al. [14] which in turn was based on Owen's [25] method for searching for GW signals from in-spiraling compact binaries. We also present some useful general properties of the metric on the signal manifold which are used in the next section. In Sec. III B we then consider the case where accurate information about one or more parameters of a NS are available (for instance through radio observations) so that the parameter space that one needs to search over is drastically reduced. We present a rigorous approach, based on differential geometric methods, to determine the exact dimensionality of the data analysis problem and formulate criteria to decide whether a given source is a one-filter target.

\section{A. Number of filters: General}

In the method presented in [14], the key idea is to first correct for the Doppler effect in the phase of the signal for 
TABLE II. The number density of filters $\rho_{N}(\mu)$, Eq. (3.1), for the circular and elliptical orbit cases (the dimensions of the parameter space are $N=3$ and $N=5$, respectively) as a function of the mismatch $\mu$.

\begin{tabular}{cccccc}
\hline \hline $\begin{array}{c}\text { Dimension of the } \\
\text { parameter space }\end{array}$ & $1 \%$ & $3 \%$ & $5 \%$ & $10 \%$ & $30 \%$ \\
\hline 3 & 649.5 & 125.0 & 58.1 & 20.5 & 3.9 \\
5 & 174963.0 & 11206.5 & 3125.0 & 552.4 & 35.4 \\
\hline \hline
\end{tabular}

each of the grid points of the parameter space and then compute the power spectrum. The latter is obtained efficiently via the FFT algorithm. Even if we know the frequency of the pulsar, it is desirable to search over a band $\simeq 1 \%$ of the pulsar frequency $[26,27]$. We therefore have a large number of frequency bins to search over and the FFT algorithm is thus computationally advantageous. If the Doppler correction is right, that is, if the signal and filter parameters match perfectly, then the signal is all concentrated at $f=f_{0}$ in the power spectrum. The grid spacing is decided by the amount the maximum of the power spectrum falls, when the parameters of the signal and filter mismatch. The mismatch $\mu$ is defined as the fractional reduction in the maximum of the power spectrum when the parameters mismatch. Fixing the mismatch $\mu$ fixes the grid spacing of the filters in the parameter space which we will denote by $\mathcal{P}$. The number density of filters (the number of filters per unit proper volume-proper volume defined through the metric) in $\mathcal{P}$ depends on $\mu$, and is denoted by $\rho_{N}(\mu)$, where $N$ is the dimension of $\mathcal{P}$. For a hyper-rectangular mesh (which does not represent necessarily the most efficient tiling of the parameter space) it reads

$$
\rho_{N}(\mu)=\left[\frac{1}{2} \sqrt{\frac{N}{\mu}}\right]^{N}
$$

In fact, the proper distance $d l$ between two filters is

$$
d l=2 \sqrt{\frac{\mu}{N}} \simeq 0.15 \sqrt{\left(\frac{\mu}{3 \%}\right)\left(\frac{5}{N}\right)} .
$$

The proper volume of $\mathcal{P}$ can be easily computed from $\gamma_{j k}$ [14]:

$$
V_{\mathcal{P}}=\int_{\mathcal{P}} d \boldsymbol{\lambda} \sqrt{\operatorname{det}|| \gamma_{j k} \|}
$$

the number of filters, $\mathcal{N}$, is then just the proper volume (3.3) times the filter density (3.1):

$$
\mathcal{N}=\rho_{N}(\mu) V_{\mathcal{P}}
$$

For the elliptical orbit we have $N=5$, while for the circular case the number reduces to $N=3$. See Table II for the relevant values of $\rho_{N}(\mu)$.

If the signal parameters are $\boldsymbol{\Lambda}=\left(f_{0}, \boldsymbol{\lambda}\right)$ and the filter parameters are $\boldsymbol{\lambda}+\Delta \boldsymbol{\lambda}$, the power spectrum for an observation time $T$ is given by

$$
P\left(f ; f_{0}, \boldsymbol{\lambda}, \Delta \boldsymbol{\lambda}\right)=\frac{\mathcal{A}^{2}}{T}\left|\int_{0}^{T} d t \exp [i \Phi(t ; \boldsymbol{\lambda}, \Delta \boldsymbol{\lambda})]\right|^{2},
$$

where

$\Phi(t ; \boldsymbol{\lambda}, \Delta \boldsymbol{\lambda})=2 \pi\left(f-f_{0}\right) t+\phi_{D}\left(t ; f_{0}, \boldsymbol{\lambda}+\Delta \boldsymbol{\lambda}\right)-\phi_{D}\left(t ; f_{0}, \boldsymbol{\lambda}\right)$.

The mismatch is both in $\boldsymbol{\lambda}$ as well as in $f$ (this can occur because of sampling at the wrong frequency) and is denoted by $m(\boldsymbol{\Lambda}, \Delta \boldsymbol{\Lambda})$ :

$$
\begin{aligned}
m(\boldsymbol{\Lambda}, \Delta \boldsymbol{\Lambda}) & \equiv 1-\frac{P\left(f ; f_{0}, \boldsymbol{\lambda}, \Delta \boldsymbol{\lambda}\right)}{P\left(f_{0} ; f_{0}, \boldsymbol{\lambda}, \overline{0}\right)} \\
& \simeq g_{\alpha \beta}(\boldsymbol{\Lambda}) \Delta \Lambda^{\alpha} \Delta \Lambda^{\beta}+o\left(\Delta \boldsymbol{\Lambda}^{3}\right)
\end{aligned}
$$

From Eqs. (3.5) and (3.7) the metric $g_{\alpha \beta}$ can be computed by Taylor expansion. It is given by

$$
g_{\alpha \beta}=\left\langle\Phi_{\alpha} \Phi_{\beta}\right\rangle-\left\langle\Phi_{\alpha}\right\rangle\left\langle\Phi_{\beta}\right\rangle
$$

where the suffix, say $\alpha$, denotes a derivative with respect to $\Delta \Lambda^{\alpha}$ and the angular brackets denote time averages defined as follows: For a function $X(t)$ defined on the data train $[0, T]$, the time average of $X$ is

$$
\langle X\rangle=\frac{1}{T} \int_{0}^{T} d t X(t)
$$

We remark that $g_{\alpha \beta}$ is not the metric which is used to calculate the proper volume, because it still includes $f$. We need to maximize over $f$, which is tantamount to projecting $g_{\alpha \beta}$ orthogonal to the $\Delta f$ direction. Thus the metric on the submanifold of the search parameters $\boldsymbol{\lambda}$ is

$$
\gamma_{j k}=g_{j k}-\frac{g_{0 j} g_{0 k}}{g_{00}} .
$$

Here the Greek and Latin indices range over the parameter $\boldsymbol{\Lambda}$ $(\alpha, \beta=0,1, \ldots, N)$ and $\boldsymbol{\lambda}(j, k=1,2, \ldots, N)$, respectively; the index 0 identifies the parameter corresponding to the frequency $f$. If $f_{\max }$ is the highest $\mathrm{GW}$ frequency that we are searching for, then we must put $f_{0}=f_{\max }$ in the above expression for $\gamma_{j k}$. The proper volume of the signal and the total number of templates can be easily derived by inserting Eqs. (3.1) and (3.10) into Eqs. (3.3) and (3.4), respectively. Notice that the parameter $\mu$ is the projected mismatch, where the maximization over $f$ has already been accounted for.

The metric defined above through the phase $\Phi$ has certain elegant properties which we will use in the next section to simplify computations:

(1) Scaling: If we scale $\Phi$ by a constant factor $\chi$, that is if we define

$$
\Phi=\chi \widetilde{\Phi}
$$

then each component of the metric $g_{\alpha \beta}$ and $\gamma_{j k}$ is scaled by the factor $\chi^{2}$ : 


$$
g_{\alpha \beta}=\chi^{2} \tilde{g}_{\alpha \beta}, \quad \gamma_{j k}=\chi^{2} \tilde{\gamma}_{j k}
$$

the determinants are scaled accordingly:

$$
\operatorname{det}|| g_{\alpha \beta}\left\|=\chi^{2 N+2} \operatorname{det}|| \tilde{g}_{\alpha \beta}\right\|, \quad \operatorname{det}|| \gamma_{j k}\left\|=\chi^{2 N} \operatorname{det}|| \tilde{\gamma}_{j k}\right\| ;
$$

the proper volume $V_{\mathcal{P}}$ is scaled by the square root of the determinant, and so is $\mathcal{N}$ :

$$
V_{\mathcal{P}}=\left(\chi^{N}\right) \tilde{V}_{\mathcal{P}}, \quad \mathcal{N}=\left(\chi^{N}\right) \tilde{\mathcal{N}} .
$$

(2) Translation: If a function only of the parameters, say $f(\boldsymbol{\Lambda})$, not containing time is added to $\Phi$, the metric remains invariant under this transformation.

These properties are easily verified. We use the first property to make all the coordinates dimensionless in the metric, compute $\widetilde{V}_{\mathcal{P}}$, the proper volume in the scaled metric, and then finally multiply by the appropriate power of $\chi$ to obtain the actual volume $V_{\mathcal{P}}$ and also the number of filters. We have already used the second property to shift the origin from the focus to the center of the elliptical orbit in Eq. (2.5). The expressions for the phases differ by an amount proportional to $a e$, which leaves the metric unaltered.

\section{B. Targeted searches}

So far we have assumed that the size of the parameter space $\mathcal{P}$ along any direction is much larger than the distance between two filters; as a consequence $\mathcal{N} \gg 1$. This is the usual situation which applies when the source parameters are not known in advance. However, about a 1000 NS's are known today - mainly in the radio band - and in looking for GW's emitted by such objects the size of $\mathcal{P}$ is drastically reduced, as some or all of the parameters are known a priori with a fair degree of accuracy.

We define

$$
\lambda_{\min }^{j} \leqslant \lambda^{j} \leqslant \lambda_{\max }^{j}, \quad j=1, \ldots, N,
$$

the parameter range over which one needs to carry out a search, so that the "error bar" is

$$
\Delta \lambda^{j}=\lambda_{\max }^{j}-\lambda_{\min }^{j} \quad j=1,2, \ldots, N
$$

We distinguish then two cases:

(i) Blind searches: here $\lambda_{\max }^{j} \gg \lambda_{\min }^{j}$ and $\Delta \lambda^{j} \sim \lambda_{\max }^{j} ; \lambda_{\max }^{j}$ and $\lambda_{\text {min }}^{j}$ are decided by the observer, based on the available theoretical understanding of the astrophysical scenario.

(ii) Targeted searches: $\lambda_{\max }^{j} \sim \lambda_{\min }^{j}$ and $\Delta \lambda^{j} / \lambda^{j}<1$ or even $\ll 1 ; \lambda_{\text {max }}^{j}$ and $\lambda_{\text {min }}^{j}$ are obtained from electromagnetic observations of the source.

In targeted searches, the analysis presented in the previous section for the estimation of the number of parameters cannot be applied directly. It must be preceded by the determination of the actual search parameters-which ones and how many-in other words the number of dimensions of the signal manifold. In fact, some of the parameters, might be known so accurately-where the required accuracy is determined by the loss of SNR that cannot exceed the maximum mismatch $\mu$-that one does not need to search through these parameters at all. Of particular interest is to determine whether any known NS is a one-filter target, that is $\mathcal{N}=1$. If this condition is satisfied, we need just one filter, constructed with the parameter values provided by the available observations.

It is easy to provide an intuitive example that shows the danger of applying the analysis of Sec. III A, without first checking the effective number of dimensions of the signal manifold. Assume, for sake of simplicity, that a generic GW signal $h$ depends only on two parameters, $\lambda^{1}$ and $\lambda^{2}$, with errors given by Eqs. (3.15) and (3.16); the metric $\gamma_{j k}$, associated with this particular waveform, is represented by a 2 $\times 2$ matrix. The number of filters given by Eqs. (3.3) and (3.4) is $\mathcal{N}\left(\Delta \lambda^{1}, \Delta \lambda^{2}\right)$, where we have explicitly included the size of the parameter space in the argument of $\mathcal{N}$. It is clear that by making, say, $\Delta \lambda^{2}$ small enough, we can always obtain $\mathcal{N}<1$. In other words our uncertainty on $\lambda^{1}$ can be arbitrarily large, but it seems that by refining our knowledge on $\lambda^{2}$ we can search for that signal with only one filter. This conclusion is clearly wrong; the correct physical interpretation is that $\lambda^{2}$ is not a search parameter, and we can assign its best value, provided by the available observations, to the entire bank of filters. However, we still need to search over $\lambda^{1}$, which will give us the requisite bank of filters. The key point is therefore to decide what is the effective number of dimensions-how many parameters one needs to search for-and then apply the analysis of the previous section to search through the appropriate parameter space.

Given any signal model $h(t ; \boldsymbol{\lambda})$ and the errors in the parameters $(3.15),(3.16)$, we provide here a criterion to decide the effective number of dimensions. We consider the generic $N$-dimensional parameter space described by the metric $\gamma_{j k}$, Eq. (3.10), where $j, k=1,2, \ldots, N$. The proper distance $d l$ between two filters is given by Eq. (3.2). The key quantity is the "thickness" $\mathcal{T}^{j}$ of the parameter space along any particular direction $\lambda^{j}$ associated with $\Delta \lambda^{j}$. From standard geometrical analysis we can derive it in a straightforward way:

$$
\mathcal{T}^{j}=\frac{\Delta \lambda^{j}}{\sqrt{\gamma^{j j}}} .
$$

Here $\gamma^{j k}$ is the inverse of $\gamma_{j k}$, which is related to $\tilde{\gamma}^{j k}$ by $\gamma^{j k}=\tilde{\gamma}^{j k} / \chi^{2} ; \sqrt{\gamma^{j j}}$ is the length of the unit vector orthogonal to the hypersurface $\lambda^{j}=$ const. The condition such that a particular $\lambda^{j}$ is not a search parameter is

$$
\max _{\boldsymbol{\lambda} \in \mathcal{P}}\left[\mathcal{T}^{j}\right] \ll d l,
$$

which, via Eqs. (3.2) and (3.17), becomes

$$
\Delta \lambda^{j} \ll 2 \sqrt{\frac{\mu}{N}} \max _{\lambda \in \mathcal{P}}\left[\sqrt{\gamma^{j j}}\right] .
$$

If the previous inequality yields for all the $N$ parameters, the source needs at most a few filters. More rigorously, for a source to be exactly a one-filter target, the diameter of the 
parameter space, namely, the maximum distance between any pair of points in $\mathcal{P}$, should be less than $d l$. The parameter space is now a $N$-dimensional parallelepiped with $2^{N}$ vertices. This yields the following condition on the parameter errors:

$$
\max \sqrt{\gamma_{j k} \Delta \lambda^{j} \Delta \lambda^{k}}<2 \sqrt{\frac{\mu}{N}}
$$

where the maximum is taken over diametrically opposite $2^{N-1}$ pairs of vertices of the parallelepiped. If Eq. (3.20) is satisfied, $\mathcal{N}=1$ and the template is constructed by setting the parameters equal to their best fit provided by the observations at hand. In Eq. (3.20) we have tacitly assumed that the size of the parallelepiped is smaller than the scale on which the manifold curves.

If $M(\leqslant N)$ parameters satisfy the condition (3.18), or equivalently, (3.19), we call $\bar{\gamma}_{j k}$ the $(N-M) \times(N-M)$ metric that describes the problem, which is constructed using the $(N-M)$ parameters that do not satisfy Eq. (3.19) in the very same way described in the previous section. The signal manifold is of a lower dimension, and the total number of filters required can be computed using Eqs. (3.3) and (3.4) for the relevant number of dimensions $(N-M)$ :

$$
\mathcal{N}_{N-M} \simeq \rho_{N-M}(\mu) \int_{\mathcal{P}_{N-M}} d V_{N-M} \sqrt{\operatorname{det}|| \bar{\gamma}_{j k} \|}
$$

Here the index $(N-M)$ in the density, the number of filters, parameter space, and volume, stresses the fact that we are considering a $(N-M)$-dimensional manifold; we also use $\simeq$ instead of "equal," because of possible edge effects, introduced by the dimensions which are close to, but not exactly, known. In the following we discuss more in detail this issue and the subtleties involved in the determination of the number of parameters.

The picture becomes complicated in the case where the thickness of the parameter space in one or more dimensions is smaller than, but of the same order of, the proper distance between two filters: $\mathcal{T}^{j} \lesssim d l$. In this case it is useful to introduce the following notation:

$$
\mathcal{T}^{j}=\delta d l, \quad 0<\delta \leqslant 1 .
$$

When $\delta \sim 1$, we must be able to reach the signal on the boundary of the parameter space, which means that the filters should be placed closer than in the case of $\delta \ll 1$. In the general case, the correct determination of the number of filters can be very complicated, due to the edge effects. For sake of simplicity, let us consider, first, the case where only one parameter, say $j=1$, satisfies Eq. (3.22). In this situation, we have

$$
(N-1)\left(\frac{d l}{2}\right)^{2}+\left(\frac{\mathcal{T}^{1}}{2}\right)^{2}=\mu
$$

Solving the above equation we have

$$
d l=2 \sqrt{\frac{\mu}{N-1}\left(1-\frac{\delta^{2}}{N}\right)},
$$

and since the unit of volume in parameter space is $d V_{\text {patch }}$ $=d l^{N-1}$, the total number of filters is given by

$$
\mathcal{N}=\rho_{N-1}(\mu) \int_{\mathcal{P}_{N-1}} d V_{N-1} \sqrt{\left(1-\frac{\delta^{2}}{N}\right)^{(1-N)} \operatorname{det}\left\|\bar{\gamma}_{j k}\right\|},
$$

where $j, k=2, \ldots, N$. It is easy to check that we recover the expected formulas for the parameter space volume from Eq. (3.25) in the limiting cases when $\delta=0,1$.

In more general cases, when two or more parameters are characterized by $\delta \leqslant 1$, the situation becomes very complex, and an "exact" determination of the number of filters particularly hard. In the case when the signal manifold is 3-dimensional and two parameters, say $\lambda_{1}$ and $\lambda_{2}$, are known with $\delta \lesssim 1$ it is still possible to obtain a rather simple expression for the thickness. In this case,

$$
\mathcal{T}=\left\{\sum_{j, k=1}^{2}\left[\left(\gamma_{j k}-\frac{\gamma_{j 3} \gamma_{k 3}}{\gamma_{33}}\right) \Delta \lambda^{j} \Delta \lambda^{k}\right]\right\}^{1 / 2} .
$$

A comparison analogous to the previous one then applies.

It is however possible to obtain an estimate of the total number of filters which is correct within a factor of a few in the general case (we will use this criterion in Sec. V, when we estimate the computational costs for known radio pulsars). We introduce the following notation: $M(\leqslant N)$ is the number of parameters that satisfy Eq. (3.22), and $M^{\prime}$ ( $\leqslant M$ ) those for which $\delta \ll 1$; the $M^{\prime}$ parameters satisfy Eq. (3.18) or (3.19), and in practice we can consider them as " "exactly" known; the value of $\delta$ that we adopt to distinguish the $M^{\prime}$ parameters from the other $M-M^{\prime}$ ones is $\delta=0.1$. Indeed, $M-M^{\prime}$ is the number of parameters for $0.1<\delta \leqslant 1$, those for which edge effects may become important. The reason for the choice $\delta=0.1$ is the following: in the case $M^{\prime}=M=1$, it is easy to verify, through Eq. (3.25), that the estimate of the total number of filters is very accurate. We assume that similar accuracy will hold also for a few number of dimensions. The values $N, M$ and $M^{\prime}$ satisfy therefore the inequality $M^{\prime} \leqslant M \leqslant N$. To summarize, we consider the $M^{\prime}$ parameters to be exactly known, $M-M^{\prime}$ ' "questionable", (border effects need to be taken into account) and $N-M$ to search for. One can imagine the questionable parameters forming a $\left(M-M^{\prime}\right)$-dimensional parallelepiped at each point of the $(N-M)$-dimensional sub-manifold. The projection of this parallelepiped "orthogonal'" to the $N-M$ dimensional sub-manifold of search parameters decides the number of filters needed for the questionable parameters. The total number of filters is therefore

$$
\mathcal{N} \lesssim 2^{\left(M-M^{\prime}\right)} \mathcal{N}_{(N-M)},
$$

where $\mathcal{N}_{(N-M)}$ is given by Eq. (3.21). The multiplicative factor $2^{\left(M-M^{\prime}\right)}$ accounts for the number of filters (which we have overestimated) to take care of each of the questionable 
parameters. Here, we have been cautious to assume two filters per parameter, say one corresponding to $\lambda_{\min }^{j}$ and one to $\lambda_{\max }^{j}$. It is simple to check that we overestimate the number of filters by applying Eq. (3.25), which is valid if $M^{\prime}=0$ and $M=1$. In the case $M-M^{\prime} \gg 1$, Eq. (3.27) might greatly overestimate the actual number of filters. In practical cases $M-M^{\prime}$ is expected to be at most a few, so that the expression (3.27) should provide a meaningful upper limit (clearly $\left.\mathcal{N}_{N-M}<\mathcal{N}<2^{\left(M-M^{\prime}\right)} \mathcal{N}_{(N-M)}\right)$; however, one can easily resolve this issue by setting up a Monte Carlo experiment to determine the additional number of filters and their location in $\mathcal{P}$ for the questionable dimensions.

\section{COMPUTATIONAL COSTS}

\section{A. Signal phase and dimensionless parameters}

It is convenient for the purposes of computation to express the phase $\Phi(t)$, Eq. (2.8), in terms of dimensionless parameters and use the above-mentioned scaling properties, Eqs. (3.11)-(3.14), to obtain the actual proper volume $V_{\mathcal{P}}$, Eq. (3.3). We write

$$
\Phi=\left(2 \pi f_{0} T\right) \widetilde{\Phi}
$$

where

$$
\widetilde{\Phi}=\kappa u+X \cos E+Y \sqrt{1-e^{2}} \sin E,
$$

and the dimensionless parameters are given by

$$
\begin{aligned}
u & \equiv \frac{t}{T}, \\
\kappa & \equiv \frac{f-f_{0}}{f_{0}}, \\
X & \equiv-a \sin \epsilon \cos \psi / c T, \\
Y & \equiv-a \sin \epsilon \sin \psi / c T, \\
\Omega & \equiv \omega T .
\end{aligned}
$$

Here $u$ is a dimensionless time satisfying $0 \leqslant u \leqslant 1$. So now the new set of parameters is $\boldsymbol{\Lambda}=(\kappa, X, Y, e, \Omega, \alpha)$ and $\boldsymbol{\lambda}$ $=(X, Y, e, \Omega, \alpha)$. The scaling factor $\chi$ in Eqs. (3.12), (3.13) and (3.14) is clearly

$$
\chi=2 \pi f_{\max } T,
$$

where we have set $f_{0}=f_{\text {max }}$, the maximum source frequency that one searches for. Using an equation analogous to Eq. (3.8) we obtain the scaled metric $\tilde{g}_{\alpha \beta}$.

The exact expression of the determinant of the metric from which we compute the volume is quite complicated. However, in this section our goal is to obtain approximate analytical expressions - which turn out to be very accurate over most of the relevant parameter range-which give us the important information about how the number of filters scales as a function of the key observables. For this purpose it is necessary to make some assumptions about the ranges of these parameters from astrophysical scenarios. The semimajor axis $a_{\mathrm{NS}}$ of a NS orbiting a companion of mass $m_{2}$ - with mass ratio $q \equiv m_{2} / m_{\mathrm{NS}}$-is

$$
\begin{aligned}
a_{\mathrm{NS}} \simeq & 2.65 \times 10^{11}\left(\frac{m_{\mathrm{NS}}}{1.4 M_{\odot}}\right)^{1 / 3}\left(\frac{\omega}{10^{-4} \mathrm{rad} / \mathrm{sec}}\right)^{-2 / 3} \\
& \times\left[\frac{q}{(1+q)^{2 / 3}}\right] \mathrm{cm}
\end{aligned}
$$

where the choice of $\omega$ corresponds to $\simeq 17 \mathrm{~h}$ orbital period (notice that for a NS in a $2 \mathrm{~h}$ orbit, the semi-major axis would be a factor $\simeq 4.2$ smaller). We take the time of observation to be typically 4 months long, so that $T=10^{7} \mathrm{sec}$ and we set, as reference, $f_{\max }=1 \mathrm{kHz}$. Thus the factor $f_{\max } T$ $\sim 10^{10}$. The dimensionless parameters $X$ and $Y$ are $X \sim Y$ $\sim a_{\mathrm{NS}} / c T$, and give essentially the size of the orbit in units of the distance light (or actually GW) travels during the observational time $T$. They are related to $\Omega$ by

$$
\begin{aligned}
\frac{a_{\mathrm{NS}}}{c T} \simeq & 8.8 \times 10^{-7}\left(\frac{m_{\mathrm{NS}}}{1.4 M_{\odot}}\right)^{1 / 3}\left(\frac{\Omega}{10^{3}}\right)^{-2 / 3}\left(\frac{T}{10^{7} \mathrm{sec}}\right)^{-1 / 3} \\
& \times\left[\frac{q}{(1+q)^{2 / 3}}\right]
\end{aligned}
$$

$$
\Omega=10^{3}\left(\frac{\omega}{10^{-4} \mathrm{rad} / \mathrm{sec}}\right)\left(\frac{T}{10^{7} \mathrm{sec}}\right)
$$

Notice that the value of $\Omega$ corresponds to an observation that extends over several orbits of the binary system. In fact $\Omega$ is the number of radians, or $\Omega / 2 \pi$ is the number of orbits, that the star completes in the time $T$. In this case, one can Taylor expand the matrix elements, and therefore the determinant, as a function of $\Omega^{-1} \ll 1$. Note, moreover, that in this case $X \sim Y \ll \Omega^{-1} \ll 1$; that is, $a$ and $\omega$ become of the same order-in geometrical units, $c=G=1$-only for a NS orbiting a companion object at a distance of the order of the gravitational radius. We will use these results in the approximation scheme, retaining only the leading order terms in $X$ and $Y$.

The other physically relevant case is in the opposite limit $\Omega \ll 1$. We therefore Taylor expand the matrix determinant as a function of $\Omega$. This case applies to observations that cover only a fraction of one orbital period, and the closed form expression that we obtain turns out to provide a rather good approximation of the full expression up to $\Omega \simeq 1$.

In the next subsection we examine the case when the orbit is circular, that is, $e=0$. We treat this simpler case first in order to obtain useful insights, before we address the general case of the elliptical orbit.

\section{B. Circular orbits}

The circular case is important both from the pedagogical and physical point of view: (a) it provides us several insights 
into the problem via a comparatively easier computation; (b) in targeted searches, several known NS's in binary systems, including Sco X-1, are essentially in a circular orbit; (c) for blind searches, present and near future processing power is likely to allow us to search over a reasonable parameter space mainly for emitters orbiting a companion with $e=0$.

For circular orbits, the expression of the phase (4.2) simplifies considerably: here $e=0$, and $\psi$ and $\alpha$ combine additively into a single parameter which we redefine again as $\alpha$, for sake of simplicity; in effect we put $\psi=0$. Then $X$ is just the projected radius of the orbit which we denote by $A$. We therefore have just 3 search parameters for which a discrete mesh of filters is required: $\boldsymbol{\Lambda}=(\kappa, A, \Omega, \alpha)$ and $\boldsymbol{\lambda}$ $=(A, \Omega, \alpha)$. The phase (4.2) becomes therefore

$$
\widetilde{\Phi}=\kappa u+A \cos (\Omega u+\alpha) .
$$

The metric $\tilde{g}_{\alpha \beta}$ [see Eq. (3.8)] is now a $4 \times 4$ matrix, and we compute it from Eq. (3.8) - with quantities scaled by the factor $2 \pi f_{0} T$, and therefore replaced with a "tilde" - and Eq. (4.12). The first derivatives are simply

$$
\begin{aligned}
& \widetilde{\Phi}_{\kappa}=u, \\
& \widetilde{\Phi}_{A}=\cos (\Omega u+\alpha), \\
& \widetilde{\Phi}_{\Omega}=-A u \sin (\Omega u+\alpha), \\
& \widetilde{\Phi}_{\alpha}=-A \sin (\Omega u+\alpha) .
\end{aligned}
$$

We need to compute the 10 time averages involving the above $\widetilde{\Phi}_{\beta}(\beta=0,1,2,3)$ and then compute the metric $\tilde{\gamma}_{j k}$ through the projection of $\tilde{g}_{\alpha \beta}$ orthogonal to $\kappa$, as discussed in Sec. III. The exact analytical expression of $\widetilde{V}_{\mathcal{P}}$ $=\sqrt{\operatorname{det}|| \tilde{\gamma}_{j k} \|}$ is very complex and not very illuminating. However, as discussed in the previous section, it is possible to compute $\operatorname{det}|| \tilde{\gamma}_{j k} \|$ in a closed form in the two relevant regimes: (i) $\Omega \gg 1$, the limit of several orbits during the observation time $T$, and (ii) $\Omega \ll 1$, the limit of monitoring a fraction of an orbit.

\section{Limit of large number of orbits}

We derive the expression of the determinant of $\tilde{\gamma}_{i j}$ in the limit $\Omega \gg 1$. The elements of $\tilde{g}_{\alpha \beta}$ and $\tilde{\gamma}_{j k}$ are explicitly given in Appendix B 1. The proper volume element reads

$$
\sqrt{\operatorname{det}|| \tilde{\gamma}_{j k} \|}=\frac{A^{2}}{\sqrt{96}}+o\left(\Omega^{-1}, A^{3}\right)
$$

Figure 2 shows the comparison of the analytical asymptotic expression (4.14) with the numerical evaluation of the full determinant. It is remarkable that for $\Omega \gtrsim 10$-which corresponds to about 2 orbits completed during $T$ - the two results are essentially identical.

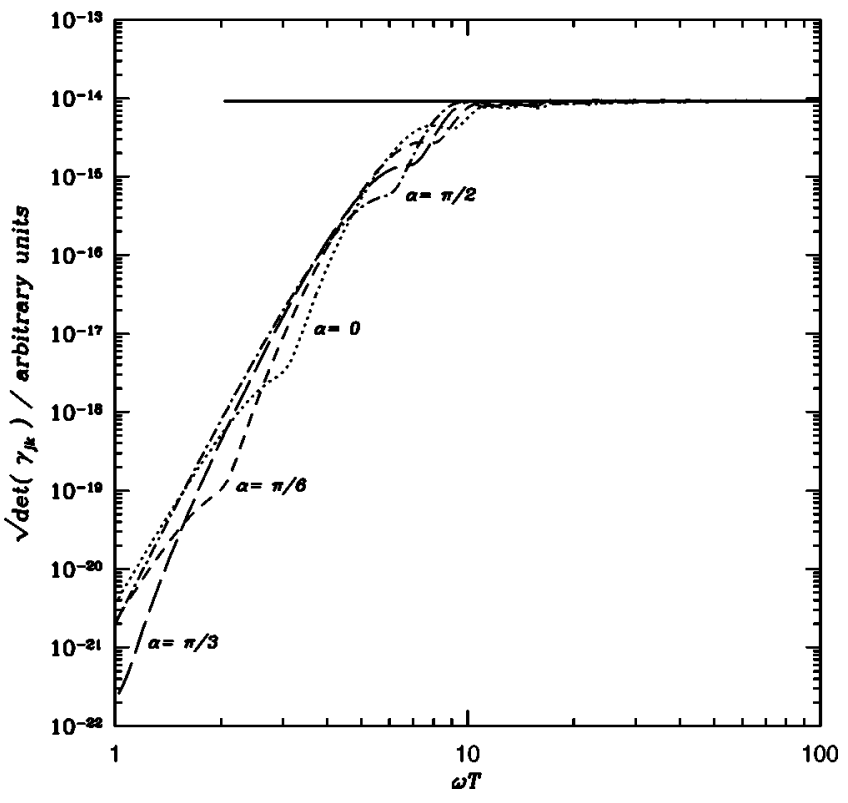

FIG. 2. Comparison of the asymptotic analytical expression and the numerical evaluation of the full determinant in the large number of orbits approximation $(\Omega \gg 1)$, for sources in circular orbits. The plot shows the proper volume element $\sqrt{\operatorname{det}\left\|\gamma_{j k}\right\|}$, in arbitrary units, as a function of the dimensionless orbital frequency parameter $\Omega$ $\equiv \omega T$. The solid line corresponds to the asymptotic expression in the regime $\Omega \gg 1$, Eq. (4.14), whereas the other curves correspond to the numerical evaluation of the full expression of $\sqrt{\operatorname{det}\left\|\gamma_{j k}\right\|}$, for several values of the initial phase of the orbit of the source: $\alpha=0$ (dotted line), $\alpha=\pi / 6$ (dashed line), $\alpha=\pi / 3$ (long-dashed line), and $\alpha=\pi / 2$ (dot-dashed line). The size of the projected semi-major axis in dimensionless units is $A=10^{-7}$. Notice that for $\Omega \geqslant 20$ the asymptotic expansion is indistinguishable from the full expression.

The proper volume $\widetilde{V}_{\mathcal{P}}$ is given by integrating the volume element (4.14) within the appropriate limits. Here we need to integrate over $A, \Omega$ and $\alpha$, within the ranges

$$
\begin{gathered}
A_{\text {min }} \leqslant A \leqslant A_{\text {max }}, \\
\Omega_{\text {min }} \leqslant \Omega \leqslant \Omega_{\text {max }}, \\
0 \leqslant \alpha \leqslant 2 \pi .
\end{gathered}
$$

Inserting Eq. (4.14) into Eq. (3.3), and taking into account the limits (4.15), we get the following proper scaled volume:

$$
\widetilde{V}_{\mathcal{P}}=\frac{\pi}{6 \sqrt{6}}\left(A_{\max }^{3}-A_{\min }^{3}\right)\left(\Omega_{\max }-\Omega_{\min }\right)
$$

Since the parameter space is 3 dimensional, $N=3$, to obtain the actual volume we need to multiply $\widetilde{V}_{\mathcal{P}}$ by the factor $\chi^{3}$ $=\left(2 \pi f_{\max } T\right)^{3}$ :

$$
V_{\mathcal{P}}=\frac{\pi}{6 \sqrt{6}}\left(\frac{2 \pi f_{\text {max }}}{c}\right)^{3}\left[a_{\mathrm{p}, \text { max }}^{3}-a_{\mathrm{p}, \text { min }}^{3}\right]\left(\omega_{\text {max }}-\omega_{\text {min }}\right) T .
$$


Notice that the factor $2 \pi f_{\max } / c$ is the maximum wave number of the gravitational wave that we want to detect. The main point in Eq. (4.17) is to observe how the volume scales. The number of filters increases linearly in the observation time $T$, so hierarchical searches based on $T$ will not work effectively (compare this to the case of the all sky all frequency searches for isolated pulsars where the patches scale as $T^{5}$ ). The volume is proportional to the cube of the size of the projected orbit along the line of sight $a_{\mathrm{p}}$. So knowing, say, the inclination angle fairly well and the radius of the orbit will greatly reduce the computational load. Similar considerations apply to the frequency.

In the case of blind searches, $\Delta \lambda^{j} \sim \lambda_{\max }^{j}$, for all the parameters. Then the parameter volume is

$$
\begin{aligned}
V_{\mathcal{P}} \simeq & 1.96 \times 10^{15}\left(\frac{f_{\max }}{1 \mathrm{kHz}}\right)^{3}\left(\frac{a_{\mathrm{p}, \max }}{10^{11} \mathrm{~cm}}\right)^{3} \\
& \times\left(\frac{\omega_{\max }}{10^{-4} \mathrm{rad} / \mathrm{sec}}\right)\left(\frac{\alpha_{\max }}{2 \pi}\right)\left(\frac{T}{10^{7} \mathrm{sec}}\right),
\end{aligned}
$$

and the total number of filters is given by

$$
\mathcal{N}(\mu=3 \%) \simeq 1.25 \times 10^{17}\left(\frac{V_{\mathcal{P}}}{10^{15}}\right) .
$$

This result indicates that the processing power to carry out a coherent blind search (even in the case of circular orbits) is outrageous; in fact, assume that the costs are entirely dominated by the computation of the FFT and the power spectrum; then, each filter, over the entire search bandwidth, requires the following number of floating point operations:

$$
\begin{aligned}
n_{\mathrm{op}} & =6 f_{\max } T\left[\log _{2}\left(2 f_{\max } T\right)+\frac{1}{2}\right] \\
& \simeq 2.1 \times 10^{12}\left(\frac{f_{\max }}{1 \mathrm{kHz}}\right)\left(\frac{T}{10^{7} \mathrm{sec}}\right) .
\end{aligned}
$$

As a consequence the total processing power or computational speed $\mathcal{S}$ to keep up with the data is

$$
\mathcal{S}=\frac{n_{\mathrm{op}} \mathcal{N}}{T} \simeq 2.1 \times 10^{16}\left(\frac{f_{\max }}{1 \mathrm{kHz}}\right)\left(\frac{\mathcal{N}}{10^{17}}\right) \text { MFlops }
$$

We would like to stress that this estimation applies only to the orbital parameters and does not take into account the costs involved in searching for the source location in the sky and the spin down parameters. We would also like to point out the very steep dependence of the processing power [cf. Eqs. (4.18) and (4.21)], on the maximum frequency $f_{\max }$ up to which the search is conducted: $\mathcal{S} \propto f_{\max }^{4}$. This indicates that restricting the frequency band translates into a major saving in computational power. As one could have imagined, from previous results pertaining to isolated sources, the additional costs to search for the orbital parameters are absolutely prohibitive. This is also due to the fact, that the acceleration of a source with an orbital companion may be much higher than detector acceleration due to Earth's rotation and orbital motion, producing a much larger frequency drift which needs to be corrected for. Clearly, only prior information on the source parameters can make such a search feasible. In fact, when some parameters are known a priori, the number of templates to process the data reduces drastically.

It is interesting to estimate the thickness of the parameter space along the different directions as a function of the parameter values and errors, in order to gain insight into the accuracy that is required to carry out a search only with a few templates. For a 3 parameter search and a mismatch $\mu$ $=3 \%$ between the signal and the template, the filter separation [see Eq. (3.2)] is $d l=0.2$; it has to be compared with the thickness $\mathcal{T}^{j}$, Eq. (3.18), for $j=1,2,3$. Using our approximate expression of the metric (cf. Appendix), we obtain

$$
\begin{array}{rl}
\mathcal{T}^{a_{\mathrm{p}}} & 1.49 \times 10^{-2}\left(\frac{\Delta a_{\mathrm{p}}}{1 \mathrm{~km}}\right)\left(\frac{f_{\max }}{1 \mathrm{kHz}}\right), \\
\mathcal{T}^{\omega} \simeq & 4.29 \times 10^{-2}\left(\frac{\Delta \omega}{10^{-12} \mathrm{sec}^{-1}}\right)\left(\frac{a_{\mathrm{p}}}{10^{11} \mathrm{~cm}}\right) \\
& \times\left(\frac{T}{10^{7} \mathrm{sec}}\right)\left(\frac{f_{\max }}{1 \mathrm{kHz}}\right), \\
\mathcal{T}^{\alpha} \simeq & 7.43 \times 10^{-2}\left(\frac{\Delta \alpha}{10^{-5} \mathrm{rad}}\right)\left(\frac{a_{\mathrm{p}}}{10^{11} \mathrm{~cm}}\right)\left(\frac{f_{\max }}{1 \mathrm{kHz}}\right) .
\end{array}
$$

For typical binary neutron stars parameters, we also show in Fig. 3 the relative errors $\Delta \lambda^{j} / \lambda^{j}$ that are required so that $\mathcal{T}^{j}<d l$. Our prior knowledge of the parameters must be very accurate in order to reduce the number of filters to only a few; typically an error $\Delta \lambda^{j} / \lambda^{j}<10^{-5}$ (or smaller) is required. However, this is not at all uncommon for radio observations and this aspect will be discussed in Sec. V.

\section{Fraction of one orbit case}

We consider now the opposite limit: $\Omega \ll 1$. This corresponds to the physical situation where pulsar radioastronomers usually apply the so-called "accelerated search" $[25,28]$, as one monitors only a small fraction of the source orbital period, say less than $1 \mathrm{rad}$. The presence of a companion object for such a short time introduces an acceleration on the source motion that could be, as first approximation, treated as constant.

For GW observations, the case $\Omega \ll 1$ is relevant for binary systems with very long orbital periods (of the order of 1 yr or more) and $T \sim 10^{7} \mathrm{sec}$ or when coherent integrations are performed on short data segments (say of the order of 1 h). The latter situation is often encountered in hierarchical data analysis schemes, where one first uses matched filters on short time base lines, and then concatenates together in a incoherent fashion the corrected data chunks.

We derive an approximate expression for the determinant of the metric $\tilde{\gamma}_{j k}$ by Taylor expanding it as a function of $\Omega$ $\ll 1$. The computation is trivial but cumbersome, and we 


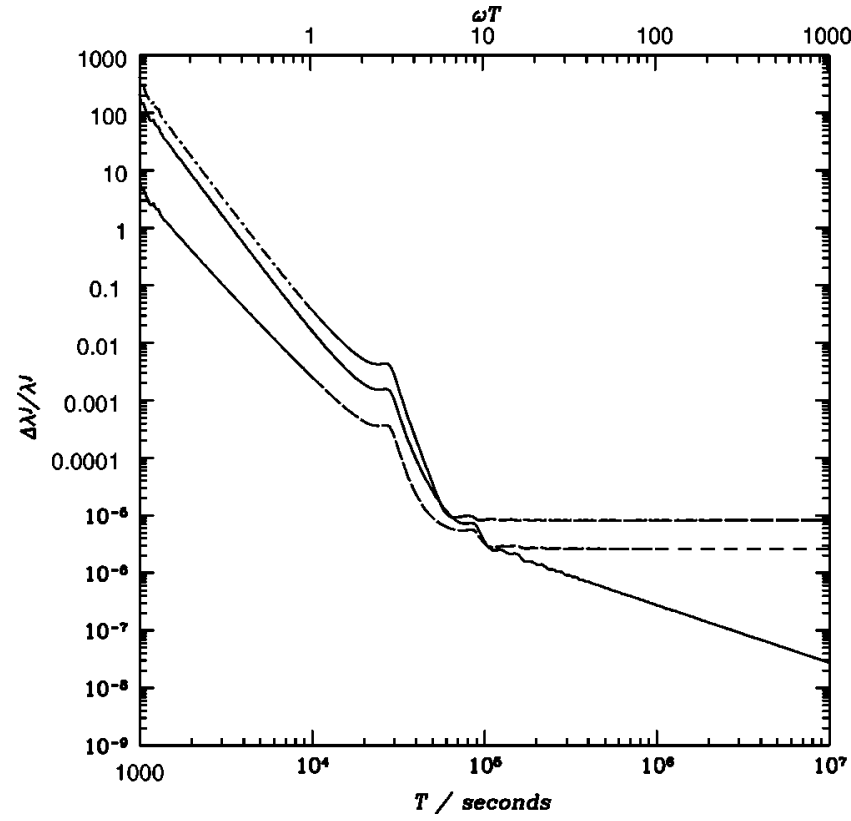

FIG. 3. The errors on the orbital parameters required to carry out a search using only a few matched filters. The relative errors in the parameters $\Delta \omega / \omega$ (solid line), $\Delta a_{\mathrm{p}} / a_{\mathrm{p}}$ (dot-dashed line) and $\Delta \alpha / \alpha$ (dashed line) are plotted for a constant thickness of $\mathcal{T}^{j}=d l$ $=0.2(N=3$ and $\mu=0.03)$; the source corresponds to a NS/NS binary system in circular orbit; the parameters have been chosen to have the following values: $\omega=1.027 \times 10^{-4} \mathrm{rad} / \mathrm{sec}, a_{\mathrm{p}}=1.64$ $\times 10^{11} \mathrm{~cm}, \alpha=0.1 \mathrm{rad}$, and $f_{\max }=1 \mathrm{kHz}$.

present the explicit expressions of the elements of the reduced metric, Eq. (3.14), with phase model (4.1) in the Appendix B 2. We obtain the following volume element:

$$
\sqrt{\operatorname{det}\left\|\gamma_{j k}\right\|}=\frac{(1+\cos 2 \alpha)^{1 / 2}}{3628800 \sqrt{70}} A^{2} \Omega^{8}+o\left(\Omega^{9}, A^{3}\right)
$$

It is interesting to note that for $\alpha=\pi / 2$ and $3 \pi / 2$ - the NS is receding from or approaching toward the detector essentially along the line of sight - the determinant tends to zero (at this order, but there are higher order corrections in $\Omega$ that depend on $\alpha$, and do not vanish): in fact the signal appears Doppler shifted by a constant indistinguishable offset, depending on the velocity of the source, which one does not need to correct for.

A comparison of the asymptotic limit (4.23) with the full expression of the determinant is shown in Fig. 4, where we present the results for two values of $\alpha=0, \pi / 3$. Notice that Eq. (4.23) is very accurate up to $\Omega \simeq 1$ for $\alpha=0$, whereas it approximates correctly the full expression only for $\Omega \lesssim 0.1$ in the case $\alpha=\pi / 3$. This is due to the fact that when $\alpha \neq 0$, the determinant contains higher order correction terms in $\Omega$, which depend on $\alpha$ and vanish for $\alpha=0$. In the range 0.1 $\lesssim \Omega \lesssim 1$ they produce a sizable effect that one cannot ignore.

By integrating Eq. (4.23) on the parameter range (4.15) and following steps similar to the previous case, we obtain the volume

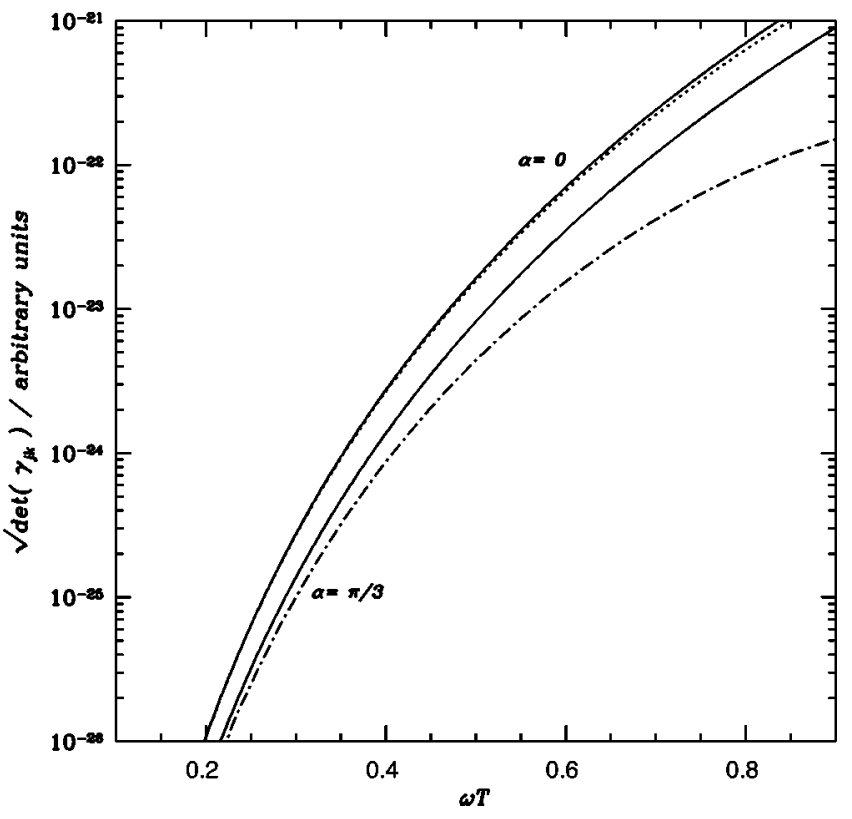

FIG. 4. Comparison of the asymptotic analytical expression and the numerical evaluation of the full determinant for the case of observations lasting a fraction of the neutron-star orbit $(\Omega \ll 1)$. The plot shows the proper volume element $\sqrt{\operatorname{det}\left\|\gamma_{j k}\right\|}$, in arbitrary units, as a function of the angular orbital frequency, in units of the observation time, $\Omega \equiv \omega T$. The solid lines correspond to the asymptotic expressions in the regime $\Omega \lesssim 1$ [see Eq. (4.23)] for selected values of the initial orbital phase of the source: $\alpha=0$ and $\pi / 3$ (see labels). For the same values of $\alpha$ we plot also $\sqrt{\operatorname{det}\left\|\gamma_{j k}\right\|}$ obtained by numerically evaluating the full expression (dotted line, $\alpha=0$; dashdotted line, $\alpha=\pi / 3)$.

$$
\begin{aligned}
V_{\mathcal{P}}= & \frac{1}{24494400 \sqrt{35}}\left(\frac{2 \pi f_{\max }}{c}\right)^{3}\left[a_{\mathrm{p}, \max }^{3}-a_{\mathrm{p}, \min }^{3}\right] \\
& \times\left[\left(\omega_{\max } T\right)^{9}-\left(\omega_{\min } T\right)^{9}\right] .
\end{aligned}
$$

Following the same scheme as in the previous section, we can obtain the total volume of the parameter space, when no prior information about the source parameters is available:

$$
\begin{aligned}
V_{\mathcal{P}} \simeq & 6.3\left(\frac{f_{\max }}{1 \mathrm{kHz}}\right)^{3}\left(\frac{a_{\mathrm{p}, \max }}{10^{11} \mathrm{~cm}}\right)^{3}\left(\frac{\omega_{\max }}{10^{-4} \mathrm{rad} / \mathrm{sec}}\right)^{9}\left(\frac{T}{1 \mathrm{~h}}\right)^{9} \simeq 3.3 \\
& \times 10^{5}\left(\frac{f_{\max }}{1 \mathrm{kHz}}\right)^{3}\left(\frac{a_{\mathrm{p}, \max }}{10^{13} \mathrm{~cm}}\right)^{3}\left(\frac{\omega_{\max }}{10^{-7} \mathrm{rad} / \mathrm{sec}}\right)^{9} \\
& \times\left(\frac{T}{1 \text { month }}\right)^{9}
\end{aligned}
$$

The total number of filters, for a mismatch of $3 \%$ and the parameters values quoted in the previous expression, is therefore $\mathcal{N} \simeq 793$ and $4.2 \times 10^{7}$ respectively. The extracomputational burden to carry out searches of NS in binaries with long orbital periods as compared with the observation time might be therefore affordable or even negligible in some cases. In fact, the processing power required to keep up with the data flow is respectively: 


$$
\mathcal{S} \simeq 1.4 \times 10^{2}\left(\frac{f_{\max }}{1 \mathrm{kHz}}\right)\left(\frac{\mathcal{N}}{10^{3}}\right) \text { MFlops } \simeq 1.9 \times 10^{6}\left(\frac{f_{\max }}{1 \mathrm{kHz}}\right)\left(\frac{\mathcal{N}}{10^{7}}\right) \text { MFlops }
$$

The costs are of course reduced if some orbital elements are known in advance, and the parameters need to be known only within a factor of $\approx 2$ to allow a few templates search. In fact, the thickness of the parameter space in the three directions becomes now

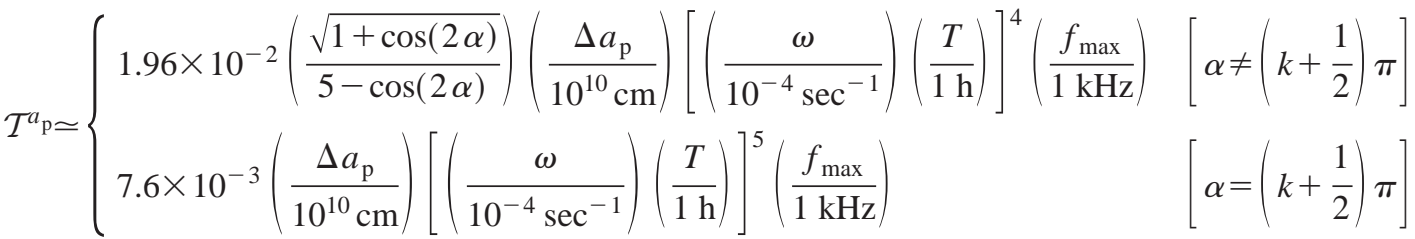

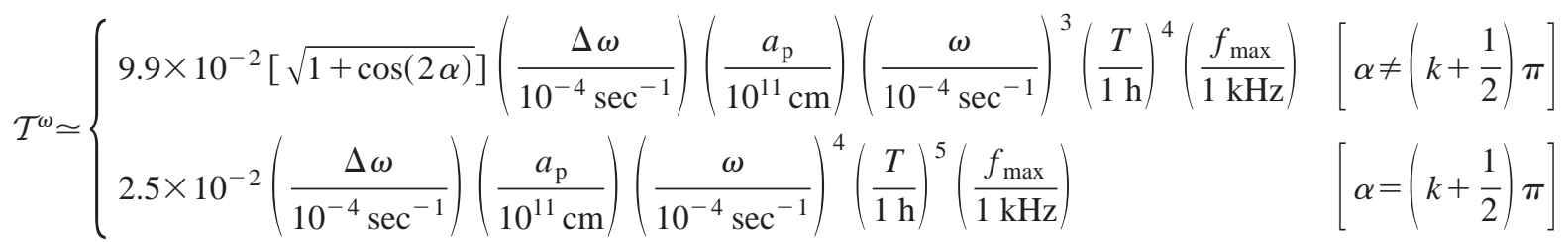

$$
\begin{aligned}
& \mathcal{T}^{\alpha} \simeq \begin{cases}2.8 \times 10^{-2} \sqrt{\frac{1+\cos (2 \alpha)}{1-\cos (4 \alpha)}}\left(\frac{\Delta \alpha}{10^{-1} \mathrm{rad}}\right)\left(\frac{a_{\mathrm{p}}}{10^{11} \mathrm{~cm}}\right)\left[\left(\frac{\omega}{10^{-4} \mathrm{sec}^{-1}}\right)\left(\frac{T}{1 \mathrm{~h}}\right)\right]^{4}\left(\frac{f_{\max }}{1 \mathrm{kHz}}\right) & {\left[\alpha \neq k \frac{\pi}{2}\right]} \\
3.8 \times 10^{-2}\left(\frac{\Delta \alpha}{10^{-1} \mathrm{rad}}\right)\left(\frac{a_{\mathrm{p}}}{10^{11} \mathrm{~cm}}\right)\left[\left(\frac{\omega}{10^{-4} \mathrm{sec}^{-1}}\right)\left(\frac{T}{1 \mathrm{~h}}\right)\right]^{3}\left(\frac{f_{\max }}{1 \mathrm{kHz}}\right) & {[\alpha=k \pi]} \\
6.3 \times 10^{-1}\left(\frac{\Delta \alpha}{10^{-1} \mathrm{rad}}\right)\left(\frac{a_{\mathrm{p}}}{10^{11} \mathrm{~cm}}\right)\left[\left(\frac{\omega}{10^{-4} \mathrm{sec}^{-1}}\right)\left(\frac{T}{1 \mathrm{~h}}\right)\right]^{2}\left(\frac{f_{\max }}{1 \mathrm{kHz}}\right) & {\left[\alpha=\left(k+\frac{1}{2}\right) \pi\right]}\end{cases}
\end{aligned}
$$

where $k$ is an integer. The difference with respect to the limit $\Omega \gg 1$ is striking. Figure 3 clearly shows the steep dependence of the thickness of $\mathcal{P}$ on $\Omega$ or, equivalently, the time of observation, where the behavior of $\Delta \lambda^{j} / \lambda^{j}$ changes abruptly when $T \sim P$. Notice also the change of scaling of the $\mathcal{T}$ s depending on the location of the source on the orbit, i.e., the value of the parameter $\alpha$.

\section{Elliptical orbits}

We have already described the orbit in Secs. II B and IV A. As a phase model we use Eq. (4.2) and take the expansion of the eccentric anomaly $E$ as a function of the mean anomaly $M$ and the eccentricity $e$ and truncate at the 7th order in $e$ [see Eqs. (2.5) and Appendix A]. This means we consider 7 harmonics in $\omega$. The volume that we obtain is also correct up to this order in $e$. The derivatives of the phase (4.2) with respect to the six parameters $\kappa, X, Y, e, \omega, \alpha$, or equivalently, $\kappa, A, e, \omega, \alpha, \psi$ are easily computed. We then proceed as in the circular orbit case, except now $\tilde{g}_{\alpha \beta}$ is a 6 $\times 6$ matrix and the $\tilde{\gamma}_{j k}$ is a $5 \times 5$ matrix. The problem is therefore much more complex than in the circular case, and it is impossible to obtain a closed form expression of the de- terminant. However, we can still Taylor-expand the relevant expressions as done before and keep the leading order terms. We present here a closed form expression of the volume element, and therefore the number of filters, in the asymptotic limit $\Omega \gg 1$. The limit $\Omega \ll 1$ can in principle be obtained by applying the same scheme adopted in the previous subsection. However, the number of terms that we need to retain is so large that the computation becomes very cumbersome. We present only the numerical results in Fig. 5, where the reader can derive the important scalings.

In the limit $\Omega \gg 1$, using the parameters $A, \psi, e, \Omega, \alpha$ as coordinates, the volume element is given by

$$
\begin{aligned}
\sqrt{\operatorname{det}\left\|\tilde{\gamma}_{j k}\right\|}= & \frac{A^{4}}{32 \sqrt{6}}\left[e-\frac{3}{4} e^{3}-\frac{41}{256} e^{5}\right. \\
& \left.+\frac{\cos 2 \psi}{32}\left(4 e^{3}-e^{5}\right)-\frac{\cos 4 \psi}{256} e^{5}\right] .
\end{aligned}
$$

Comparing the former expression with the numerical evaluation of the full determinant (see Fig. 5), it turns out-as it 


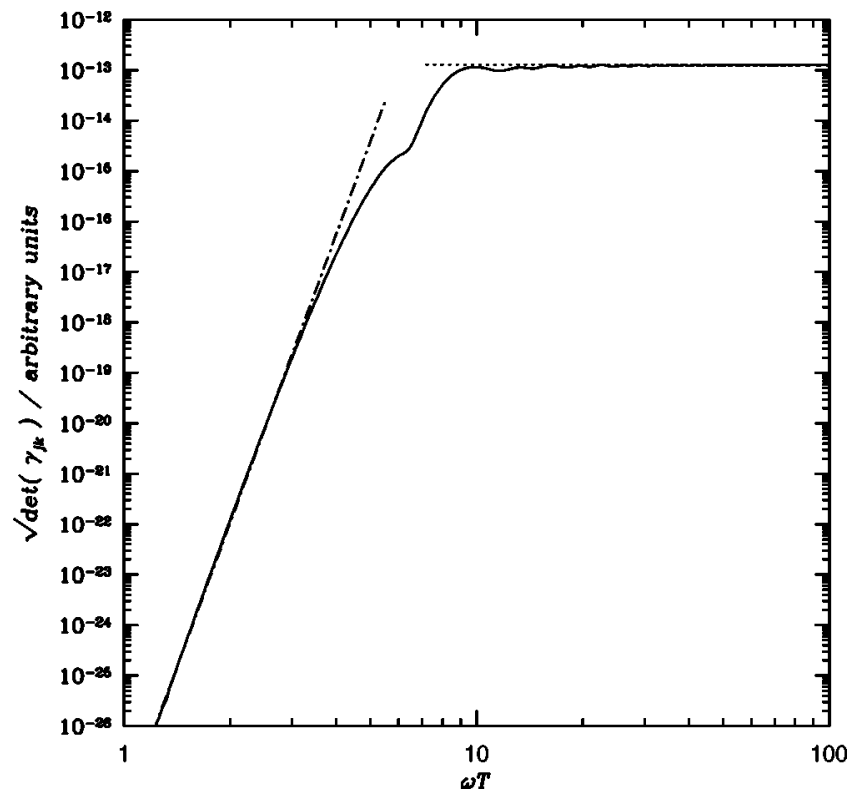

FIG. 5. Comparison of the asymptotic analytical expression and the numerical evaluation of the proper volume element of the signal manifold for neutron stars in elliptical orbits. The plot shows $\sqrt{\operatorname{det}\left\|\gamma_{j k}\right\|}$, in arbitrary units, as a function of the dimensionless orbital frequency parameter $\Omega \equiv \omega T$. The solid line corresponds to the exact expression of the determinant, computed numerically. The dotted line refers to the asymptotic expression of the determinant [cf. Eq. (4.28)] in the regime $\Omega \gg 1$; the dot-dashed line is the asymptotic expression of the determinant for $\Omega \ll 1$, in which case $\sqrt{\operatorname{det}\left\|\tilde{\gamma}_{j k}\right\|} \propto \Omega^{19}$. The size of the projected semi-major axis in dimensionless units is $A=10^{-7}$; the other orbital elements are chosen as $e=0.5$ and $\alpha=\psi=0$.

happened for $e=0$ (cf. the previous sections)-that Eq. (4.28) is accurate within a few percent even if the number of orbits completed during the time $T$ is just about 2 or 3 .

In order to obtain the proper volume of the parameter space, we integrate Eq. (4.28) over the parameter range:

$$
\begin{gathered}
A_{\min } \leqslant A \leqslant A_{\max }, \\
\Omega_{\min } \leqslant \Omega \leqslant \Omega_{\max }, \\
0 \leqslant e \leqslant e_{\max }, \\
0 \leqslant \alpha \leqslant 2 \pi, \\
0 \leqslant \psi \leqslant 2 \pi,
\end{gathered}
$$

and multiply by the scaling factor $\chi^{5}=\left(2 \pi f_{\max } T\right)^{5}$. We have the following expression:

$$
\begin{aligned}
V_{\mathcal{P}}= & \frac{\pi^{2}}{160 \sqrt{6}} F\left(e_{\max }\right)\left(\frac{2 \pi f_{\text {max }}}{c}\right)^{5} \\
& \times\left[a_{\mathrm{p}, \text { max }}^{5}-a_{\mathrm{p}, \text { min }}^{5}\right]\left(\omega_{\max }-\omega_{\min }\right) T,
\end{aligned}
$$

where

$$
F(e)=e^{2}\left(1-\frac{3}{8} e^{2}-\frac{41}{768} e^{4}\right)
$$

We can check how accurate this expression is by switching off the $e^{6}$ term in Eq. (4.31) and comparing with the full one up to $o\left(e^{6}\right)$. We find that the expressions agree very well to about $e \sim 0.7$. From this we surmise that the Eqs. (4.30) and (4.31) will be correct up to $e \sim 0.8$. We also checked how well the leading order term $e^{2}$ approximates $F(e)$, and we found that up to $e=0.5$ and $e=0.8$ (as reference values), they agree within $\simeq 10 \%$ and $\simeq 35 \%$, respectively.

For blind searches, the size of the parameter space is

$$
\begin{aligned}
V_{\mathcal{P}} \simeq & 2.3 \times 10^{22}\left(\frac{f_{\max }}{1 \mathrm{kHz}}\right)^{5}\left(\frac{a_{\mathrm{p}, \max }}{10^{11} \mathrm{~cm}}\right)^{5} \\
& \times\left(\frac{\omega_{\max }}{10^{-4} \mathrm{rad} / \mathrm{sec}}\right)\left(\frac{T}{10^{7} \mathrm{sec}}\right),
\end{aligned}
$$

where we have evaluated Eq. (4.30) for $e_{\max }=0.5$. For $e_{\max }$ $=0.1$ and $e_{\max }=0.8$ the volume changes by the factors $\simeq 0.04$ and $\simeq 2$, respectively. The total number of filters now is

$$
\mathcal{N}(\mu=3 \%) \simeq 1.1 \times 10^{26}\left(\frac{V_{\mathcal{P}}}{10^{22}}\right)
$$

Indeed, for $e \neq 0$, prior information about the source parameters is essential in order to reduce the computational costs; see Eq. (4.21). In particular the steep dependence on $a_{\mathrm{p}}$ and $f$, both to the fifth power, indicates that the computational load could be cut down substantially if we knew them fairly accurately. These are the crucial parameters which govern the computational costs. $\mathcal{N}$ still scales linearly with $\omega T$. For sufficiently small values of the eccentricity, it scales quadratically with $e$.

As we did in Sec. IV B, we can estimate the thickness of the parameter space along a given direction and compare it to the filter separation $d l$, which for $N=5$ and $\mu=3 \%$ is $\simeq 0.15$ [see Eq. (3.2)]. This is useful in obtaining insights into the accuracy needed in the parameters for reducing the computational costs by a large factor, so that they become affordable. In Fig. 6 we show the relative errors $\Delta \lambda^{j} / \lambda^{j}$, for typical orbital elements of a NS/NS binary, as a function of the observation time, where we set $\mathcal{T}^{j}=d l$. For observation times involving several orbits, $\Omega \gg 1$, one needs to know a parameter with an accuracy of the order of 1 part in a $10^{6}$, in order that the search be carried out with the help of only a few filters. However, as in the circular orbit case, $\Delta \lambda^{j} / \lambda^{j}$ increases very steeply when $T$ becomes of the order of $P$, or 


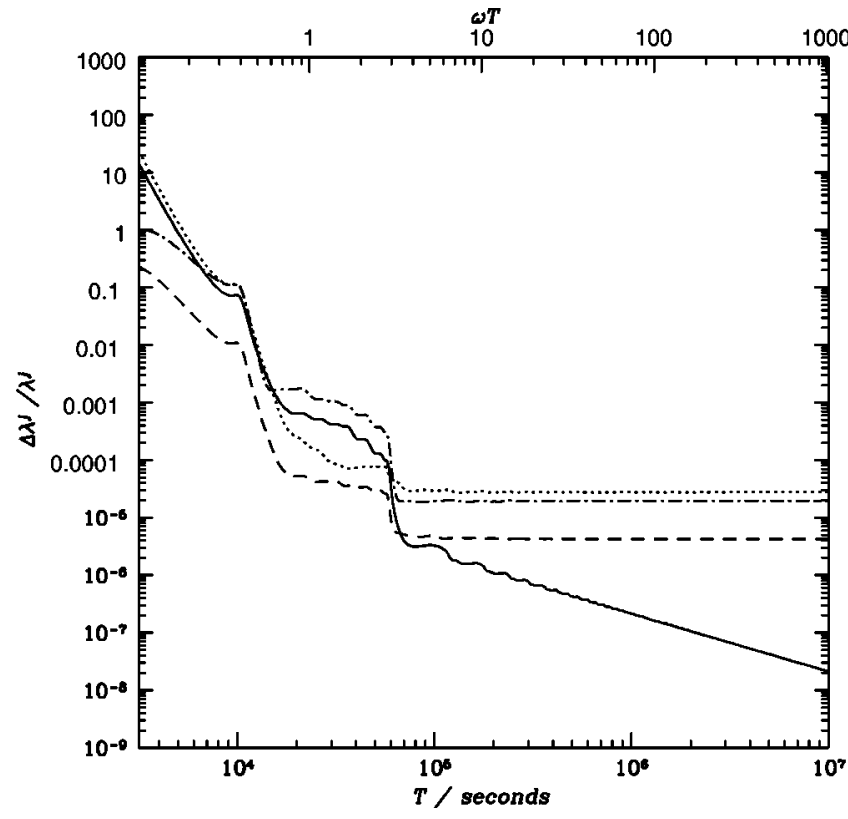

FIG. 6. The errors in the orbital parameters required to carry out a search using only a few matched filters for a binary in eccentric orbit. The relative errors in the parameters $\Delta \omega / \omega$ (solid line), $\Delta X / X$ (dot-dashed line), $\Delta e / e$ (dotted line) and $\Delta \alpha / \alpha$ (dashed line) are plotted for constant thickness equal to the filter separation $d l$ $\simeq 0.15$, corresponding to $\mu=0.03$, for an edge-on eccentric orbit with parameters: $\omega=1.027 \times 10^{-4} \mathrm{rad} / \mathrm{sec}, a_{\mathrm{p}}=1.64 \times 10^{11} \mathrm{~cm}, e$ $=0.5$.

shorter. In this case a $1 \%$ accuracy (compare it to the case $e=0$, where $\Delta \lambda^{j} / \lambda^{j} \sim 2$ ) is sufficient to carry out the search with few filters.

\section{COMPUTATIONAL COSTS FOR KNOWN NEUTRON STARS}

In the previous sections we have analyzed the extra computational costs required in correcting for the Doppler effect produced by the motion of a NS around a companion object, on a monochromatic GW signal. We are therefore in a position to apply the former results to known neutron stars which are amenable to searches by Earth-based detectors. Here we deal with two main classes of sources: the NS's known as radio pulsars and the NS's in LMXB's, in particular Sco $\mathrm{X}-1$.

\section{A. Radio pulsars}

The last published version of the pulsar catalog [17] contains 706 NS's, of which 44 are in binary systems. Actually, the total number of pulsars known today is $\sim 1200$, thanks mainly to the recent multi-beam survey of the southern sky carried out at the Parkes radio telescope in New South Wales, Australia [29-31]. A further considerable increase in the number of pulsars is expected over the next few years when the upgrade of other radio telescopes will be completed, allowing a survey of the northern sky similar to the
TABLE III. The number of filters required to search for NS's known as radio pulsars in binaries. We list the total number of filters $\mathcal{N}$ required to search for the 44 NS's in binary system contained in the catalog Taylor et al. [17] for an observation time $T$ $=1 \mathrm{yr}$ and a maximum mismatch of $3 \%$.

\begin{tabular}{|c|c|c|}
\hline PSR J & PSR B & $\mathcal{N}$ \\
\hline 0024-7204E & $0021-72 \mathrm{E}$ & $1.8 \times 10^{3}$ \\
\hline 0024-7204I & 0021-72I & $4.1 \times 10^{6}$ \\
\hline 0024-7204J & $0021-72 J$ & $9.2 \times 10^{2}$ \\
\hline $0034-0534$ & & 1. \\
\hline 0045-7319 & & signal outside the sensitivity window \\
\hline $0218+4232$ & & $1.5 \times 10^{2}$ \\
\hline $0437-4715$ & & 1 \\
\hline 0613-0200 & & 1 \\
\hline $0700+6418$ & $0655+64$ & 1 \\
\hline $0751+1807$ & & $1.1 \times 10^{7}$ \\
\hline $0823+0159$ & $0820+02$ & signal outside the sensitivity window \\
\hline $1012+5307$ & & 4 \\
\hline $1022+10$ & & 2 \\
\hline $1045-4509$ & & 16 \\
\hline $1300+1240$ & $1257+12$ & 1 \\
\hline $1302-6350$ & $1259-63$ & 2 \\
\hline $1312+1810$ & $1310+18$ & $3.9 \times 10^{9}$ \\
\hline $1455-3330$ & & 2.6 \\
\hline $1518+4904$ & & 2 \\
\hline $1537+1155$ & $1534+12$ & 1 \\
\hline $1623-2631$ & $1620-26$ & 8 \\
\hline $1640+2224$ & & 4 \\
\hline $1641+3627 \mathrm{~B}$ & $1639+36 \mathrm{~B}$ & $6.4 \times 10^{3}$ \\
\hline $1643-1224$ & & $7.3 \times 10^{4}$ \\
\hline $1713+0747$ & & 1 \\
\hline $1721-1936$ & $1718-19$ & signal outside the sensitivity window \\
\hline 1748-2446A & $1744-24 \mathrm{~A}$ & 4 \\
\hline $1803-2712$ & $1800-27$ & signal outside the sensitivity window \\
\hline $1804-0735$ & $1802-07$ & 4 \\
\hline $1804-2718$ & & $1.6 \times 10^{7}$ \\
\hline $1823-1115$ & $1820-11$ & signal outside the sensitivity window \\
\hline $1834-0010$ & $1831-00$ & signal outside the sensitivity window \\
\hline $1857+0943$ & $1855+09$ & 1 \\
\hline $1910+0004$ & & $1.2 \times 10^{2}$ \\
\hline $1915+1606$ & $1913+16$ & 1 \\
\hline $1955+2908$ & $1953+29$ & 4 \\
\hline $1959+2048$ & $1957+20$ & 2 \\
\hline $2019+2425$ & & 1 \\
\hline $2033+17$ & & $8.9 \times 10^{10}$ \\
\hline $2130+1210 \mathrm{C}$ & $2127+11 C$ & 33 \\
\hline $2145-0750$ & & 4 \\
\hline $2229+2643$ & & 16 \\
\hline $2305+4707$ & $2303+46$ & signal outside the sensitivity window \\
\hline $2317+1439$ & & 1 \\
\hline
\end{tabular}

one carried out at Parkes. However, the newly discovered sources have not yet been included into a publicly available database, and we therefore restrict ourselves to the catalog of 1995. Clearly, when the newly discovered NS's and their 
fundamental parameters will be available, our present analysis can be applied to the new objects in a straightforward way [32].

In order to compute the number of filters required to target the 44 NS's, we apply the scheme presented in the previous sections. More specifically, we use the full and exact expression of the parameter space volume computed numerically, with the values of the parameters and errors quoted in [17]; the fundamental ones are also shown in Table I. For several sources the orbital parameters are known very accurately, so that we find that the search is limited either over a small region of the parameter space or to a smaller number of dimensions $(<5)$, or both. We first estimate the actual number of dimensions of the signal manifold, following the discussion in Sec. III B, and then calculate the total number of filters from Eq. (3.27). We set the value of $\delta=0.1$ - the thickness of the parameter space in units of the filter distance [see Eq. (3.22)] —which distinguishes essentially the known parameters from the questionable ones.

The results of our analysis are presented in Table III, which lists the number of templates required for an integration time of $T=1 \mathrm{yr}$. The key point to notice is that the most interesting NS's require at most a few filters in correcting for the orbital motion: J0437-4715: NS/white dwarf system; J0437-4715: NS/white dwarf system; B1257+12: NS/planets system; J1537+1155: NS/NS binary; J1623-2631: NS/white dwarf system; and J1300+1240: NS/main sequence star binary. The upper limits on $h_{\mathrm{c}}$ are, in fact, within the sensitivity of the first or second generation of detectors; cf. Fig. 1. The data analysis costs for such sources are therefore minimal. Also considering that the frequency is very accurately known within a small bandwidth, one can substantially down-sample the data stream around the expected source frequency. Moreover, the orbital companions of the above-mentioned five neutron stars are also very different, which boosts the astrophysical interest in monitoring them.

On the other hand, for those NS's about which no information about the spin down is available, we cannot set upper limits on the signal strain and hence the computational costs become considerable. The number of filters is in the range $\sim 10^{2}-10^{10}$, for a year's worth of integration time. Therefore, even assuming a search in a narrow frequency range, around twice the radio frequency, the computational burden is overwhelming. The situation is made worse by the lack of information about $\dot{f}$ and higher time derivatives of the frequency, so that one would also have to scan the space of spin-down parameters, increasing the costs further. It is clear that more accurate data from radio observations are necessary in order to narrow down the uncertainty in the source parameters for making such searches feasible.

To summarize, the interesting radio pulsars in binary systems do not lead to large extra computational load when correcting for the orbital parameters of the source. On the other hand, for the NS's for which we have no idea about the GW strain amplitude, the computational costs are not affordable. Such sources call for a follow-up of radio observations for obtaining more accurate measurements of the orbital parameters.

\section{B. Sco X-1}

Low mass x-ray binaries could be primary GW sources for Earth-based detectors (see discussion in the Introduction). Our current astrophysical understanding and signal modeling suggests that at least one system, namely, Sco $\mathrm{X}-1$ - the brightest $\mathrm{x}$-ray source in the sky, located at a distance $2.8 \pm 0.3 \mathrm{kpc}$ - is just within the reach of the first generation of detectors with very long coherent observation times (of the order of $2 \mathrm{yr}$ ), and clearly detectable by the second generation of instruments (in particular LIGO II, in narrow-band configuration), for an integration time as little as $\approx 15$ days.

Sco X-1 orbits a low-mass companion in an essentially circular orbit with a period $P=0.787313$ (1) days; the orbital angular velocity is $\omega \simeq 9.24 \times 10^{-5} \mathrm{rad} / \mathrm{sec}$ and is known with an error $\Delta \omega=2.34 \times 10^{-10} \mathrm{rad} / \mathrm{sec}$. The radial component of the orbital velocity is $v_{\mathrm{r}}=58.2(3.0) \mathrm{km} / \mathrm{sec}$ [33]; therefore the projection of the semi-major axis along the line of sight is $a_{\mathrm{p}}=v_{\mathrm{r}} / \omega \simeq 6.3 \times 10^{10} \mathrm{~cm}$. The relative error on the semi-major axis is $\Delta a_{\mathrm{p}} / a_{\mathrm{p}}=\Delta P / P+\Delta v_{\mathrm{r}} / v_{\mathrm{r}}$ $\simeq \Delta v_{\mathrm{r}} / v_{\mathrm{r}}$, since the period is very accurately measured. This yields an error $\Delta a_{\mathrm{p}} \simeq 6.5 \times 10^{9} \mathrm{~cm}$. The position of the NS in the orbit is known with an error $\Delta \alpha=0.2 \mathrm{rad}$ [34]. The GW emission frequency is not very well determined; conservatively we assume it to be within the band $500 \mathrm{~Hz}-600 \mathrm{~Hz}$, and therefore we take the bandwidth $\Delta f \approx 100 \mathrm{~Hz}$ [20]. In the expressions for thicknesses of the parameter space below, it is appropriate to replace $f_{\max }$ by the bandwidth $\Delta f$ over which the search would be launched.

The main problems that one faces in detecting (or setting upper limits on) GW's emitted by Sco X-1 are: (i) the drift in the GW frequency, which is not likely to be monotonic, and is related to the time-varying accretion rate which is ultimately responsible for the net quadrupole moment, and (ii) the need for taking into account the Doppler phase modulation caused by the orbital motion. Present order-ofmagnitude estimates of the frequency evolution suggest that for a time duration of $\approx 10$ days the signal is confined within a single frequency bin; it determines the longest coherent integration time (or base-line duration of observations) that one can employ in searching for a monochromatic signal. As a conservative limit, we take 1 week: $T=6.05 \times 10^{5} \mathrm{sec}$. Notice that during this time the radiation is monochromatic, but emitted in an unknown frequency bin inside the band 500 $\mathrm{Hz}-600 \mathrm{~Hz}$; the position of the source is also known very accurately, so that one is essentially left with the orbital parameters that one must search over. The estimate of the computational costs that we present therefore reflects the total costs involved in searching for Sco X-1.

It is interesting to consider first the thickness of the parameter space and the conditions such that Sco X-1 is a onefilter target. Notice first that $\Omega=1$ for $T=1.08 \times 10^{4} \mathrm{sec}$ $=3.01 \mathrm{~h}$. In the limit $\Omega \ll 1$, therefore up to $\simeq 3 \mathrm{~h}$, we have 


$$
\begin{aligned}
\mathcal{T}^{a} \simeq & 9.3 \times 10^{-4}\left(\frac{\sqrt{1+\cos (2 \alpha)}}{5-\cos (2 \alpha)}\right)\left(\frac{\Delta a_{\mathrm{p}}}{6.5 \times 10^{9} \mathrm{~cm}}\right) \\
& \times\left[\left(\frac{\omega}{9.24 \times 10^{-5} \mathrm{sec}^{-1}}\right)\left(\frac{T}{1 \mathrm{~h}}\right)\right]^{4}\left(\frac{\Delta f}{100 \mathrm{~Hz}}\right), \\
\mathcal{T}^{\omega} \simeq & 1.6 \times 10^{-8}[\sqrt{1+\cos (2 \alpha)}]\left(\frac{\Delta \omega}{2.34 \times 10^{-10} \mathrm{sec}^{-1}}\right) \\
& \times\left(\frac{a_{\mathrm{p}}}{6.3 \times 10^{10} \mathrm{~cm}}\right)\left(\frac{\omega}{9.24 \times 10^{-5} \mathrm{sec}^{-1}}\right)^{3} \\
& \times\left(\frac{T}{1 \mathrm{~h}}\right)^{4}\left(\frac{\Delta f}{100 \mathrm{~Hz}}\right), \\
\mathcal{T}^{\alpha} \simeq & 2.6 \times 10^{-3} \sqrt{\frac{1+\cos (2 \alpha)}{1-\cos (4 \alpha)}}\left(\frac{\Delta \alpha}{0.2 \mathrm{rad}}\right)\left(\frac{a_{\mathrm{p}}}{6.3 \times 10^{10} \mathrm{~cm}}\right) \\
& \left.\times\left(\frac{\omega}{9.24 \times 10^{-5} \mathrm{sec}^{-1}}\right)\left(\frac{T}{1 \mathrm{~h}}\right)\right]^{4}\left(\frac{\Delta f}{100 \mathrm{~Hz}}\right) .
\end{aligned}
$$

Notice that the expressions for $\mathcal{T}^{a} \mathrm{p}$ and $\mathcal{T}^{\omega}$ are given for $\alpha$ $\neq k+\pi / 2$, where $k$ is an integer; the expression for $\mathcal{T}^{\alpha}$ is valid if $\alpha \neq k \pi / 2$. In the other cases one can easily derive the value of the thickness by applying the appropriate formula, as given in Eq. (4.27).

If one takes a 1-h-long data stretch, Sco X-1 in a one-filter target. For longer observational times up to $T \approx 3 \mathrm{~h}$, the search requires at most a few templates to take care of "border effects.' A coherent search over a bandwidth $\approx 100 \mathrm{~Hz}$ can be performed very inexpensively, and one needs a fraction of an MFlop to keep up with the data flow, which can be easily derived from Eq. (4.21) with $f_{\max }$ replaced by $\Delta f$ $\approx 100 \mathrm{~Hz}$. However, the computational cost increases steeply (as shown in the previous section) when $T$ becomes of the order of the orbital period or longer. In fact, for $\Omega \gg 1$, the thickness of the parameter space becomes

$$
\begin{array}{rl}
\mathcal{T}^{a} \mathrm{p} & 96.8\left(\frac{\Delta a_{\mathrm{p}}}{6.5 \times 10^{9} \mathrm{~cm}}\right)\left(\frac{\Delta f}{100 \mathrm{~Hz}}\right) \\
\mathcal{T}^{\omega} \simeq & 6.3 \times 10^{-3}\left(\frac{\Delta \omega}{2.3 \times 10^{-10} \mathrm{sec}^{-1}}\right)\left(\frac{a_{\mathrm{p}}}{6.3 \times 10^{10} \mathrm{~cm}}\right) \\
& \times\left(\frac{T}{10^{5} \mathrm{sec}}\right)\left(\frac{\Delta f}{100 \mathrm{~Hz}}\right) \\
\mathcal{T}^{\alpha} \simeq & 93.6\left(\frac{\Delta \alpha}{0.2 \mathrm{rad}}\right)\left(\frac{a_{\mathrm{p}}}{6.3 \times 10^{10} \mathrm{~cm}}\right)\left(\frac{\Delta f}{100 \mathrm{~Hz}}\right)
\end{array}
$$

One needs a mesh of filters for $a_{\mathrm{p}}$ and $\alpha$, but up to $T \approx 1$ month the orbital period is not a search parameter (one might actually need up to 2 filters to take care of the errors in $P$ ). The reduced metric $\bar{\gamma}_{j k}$ which we need to consider is given by a $2 \times 2$ matrix on $a_{\mathrm{p}}$ and $\alpha$. By applying Eq. (3.25), with $\mu=0.03$ and $T \lesssim 1$ month, we obtain

$$
\mathcal{N}_{(N=2)} \simeq 5.2 \times 10^{6}\left[1-\left(\frac{\delta^{2}}{3}\right)\right]^{-1} .
$$

A 1 week coherent search involving $5 \times 10^{6}$ filters requires $\approx 84$ GFlops of processing power to keep up with the data flow. For longer integration times $T \gtrsim 1$ month, $\omega$ becomes a search parameter; notice that in this case the signal is not longer monochromatic, and one needs to search also over the spin-down parameter space (which is not trivial, due to our poor understanding of the accretion rate and angular momentum transfer; see Sec. I B); the number of filters to correct only for the Doppler shift induced by Sco X-1 orbital motion is given now by

$$
\mathcal{N}_{(N=3)} \simeq 3.1 \times 10^{8}\left(\frac{T}{10^{7} \mathrm{sec}}\right) .
$$

By setting a coarser grid, increasing the mismatch from, say, $3 \%$ to $30 \%$, will reduce the number of filters in the two cases (5.3) and (5.4) by a factor of $\simeq 10$ and $\simeq 30$, respectively. The values still indicate that the computational costs are too high which is also due to the large frequency band that one needs to search through. These results clearly show that it is mandatory to determine the orbital parameters more accurately in order to reduce the parameter volume so that Sco X-1 can be monitored continuously. Equation (5.2) implies that the errors on $a_{\mathrm{p}}$ and $\alpha$ must be reduced by 3 orders of magnitude, so that only a few templates are required for the search. Although this calls for a very large observational step, it is still possible.

\section{CONCLUSIONS}

We have estimated the extra-computational costs required to search for cw sources in binary systems. This work was motivated by the fact that LMXB's, a key class of sources, possibly, for the initial generation of detectors and certainly for the enhanced detectors, are NS's orbiting a binary companion. Also several NS's detected in the radio band are in binaries and will be continously monitored by the Earthbased network of GW detectors. In order to disentangle the computational costs arising from correcting for the Doppler phase modulation produced by the source motion around the orbital companion, we have assumed that the signal is exactly monochromatic and the location of the source in the sky is perfectly known. Our results provide an estimate of the computational burden associated with these extra dimensions of the parameter space which has been neglected so far (for rather obvious reasons). We have deduced closed form analytical expressions for the number of templates required to carry out the analysis. We have obtained the scaling of the computational costs as a function of the orbital elements and the observational parameters (the source emission frequency and the observation time); they are ready-to-use tools to evaluate the computational resources needed and the trade off for search strategies. We have applied our analysis to known radio pulsars and Sco X-1, estimating the computational costs involved in targeting these systems. The analysis also addresses the need of further astronomical observations 
of known NS's, with the aim of reducing the errors in the source parameters, so that the search is made inexpensive. In fact, a by-product of our analysis is the rigorous formulation, in the geometrical framework of data analysis, of the conditions, i.e. the size of the parameter space, such that a NS can be considered a one-filter target; our result is completely general, and can be applied to any class of sources (not necessarily cw sources) whose waveforms can be modeled reliably.

Our analysis is clearly limited in several respects. Possibly, the main accomplishment is a quantitative understanding of the key issues that require intensive attack, and identifying the bottlenecks that prevent us from carrying out blind searches of NS in binary systems. There are three clear directions of future work that we believe should be pursued vigorously, particularly in the light of the current data analysis effort in setting up GW search codes:

(1) The software implementation of matched filter based searches of a NS in a binary system, for given values of the source parameters such as location in the sky, spin down(s), orbital elements. This task is simple in principle, as the fundamental building blocks are already available: search codes for GW's from isolated NS's and publicly available software (such as TEMPO) in the radio pulsar community to correct for the Doppler effect of the signal phase for a source in a binary system. However, the implementation of this might not prove to be so trivial.

(2) The estimation of the computational costs keeping into account both the orbital elements and the the source position and the spin-down parameters. This is the composite problem where the full expression for the total costs needs to be obtained. It will take into account possible correlations between the search parameters investigated here and those that describe isolated neutron stars.

(3) The investigation of suitable hierarchical strategies that can reduce the computational costs as compared to fully coherent searches. The current vigorous effort in the study and software implementation of hierarchical algorithms for isolated sources, the so-called stack-and-slide and Houghpattern-tracking searches, provides already a template for investigations for a larger parameter space. Moreover, radio and $\mathrm{x}$-ray astronomers are currently investigating algorithms suitable to carry out analogous searches in the electromagnetic spectrum [35,36].

One limitation of our approach is that we have completely neglected relativistic effects in the model of a binary orbit. In particular, the advance of the periapse is very significant in relativistic systems (the largest periapse advance reported in [17] is $\approx 4 \mathrm{deg} / \mathrm{yr}$ ). This and other complications with respect to the simple Keplerian model that we have adopted here need to be taken into account in future studies and addressed carefully.

We would like to conclude by stressing that the data analysis problem that we have addressed here is common to electro-magnetic observations of pulsating stars in binary orbits, where typical examples are radio and x-ray binaries. We can therefore hope that the current efforts in other related research fields can help in providing a breakthrough in this area.

\section{ACKNOWLEDGMENTS}

We would like to thank C. Cutler, M. A. Papa and B. Schutz for helpful discussions.

\section{APPENDIX A: COEFFICIENTS OF THE TAYLOR EXPANSION OF THE ECCENTRIC ANOMALY}

Here we give the expressions of the coefficients $\mathcal{C}_{k}(e)$ and $\mathcal{S}_{k}(e)$ which appear in the expressions for $\cos E$ and $\sqrt{1-e^{2}} \sin E$ in Eq. (2.9), upto the 7th order in $e$ [22]:

$$
\begin{aligned}
& \mathcal{C}_{0}=-\frac{1}{2} e, \\
& \mathcal{C}_{1}=1-\frac{3}{8} e^{2}+\frac{5}{192} e^{4}-\frac{7}{9216} e^{6}, \\
& \mathcal{C}_{2}=\frac{1}{2} e-\frac{1}{3} e^{3}+\frac{1}{16} e^{5}, \\
& \mathcal{C}_{3}=\frac{3}{8} e^{2}-\frac{45}{128} e^{4}+\frac{567}{5120} e^{6}, \\
& \mathcal{C}_{4}=\frac{1}{3} e^{3}-\frac{2}{5} e^{5}, \\
& \mathcal{C}_{5}=\frac{125}{384} e^{4}-\frac{4375}{9216} e^{6}, \\
& \mathcal{C}_{6}=\frac{27}{80} e^{5}, \\
& \mathcal{C}_{7}=\frac{16807}{46080} e^{6} \\
& \mathcal{S}_{1}=1-\frac{5}{8} e^{2}-\frac{11}{192} e^{4}-\frac{457}{9216} e^{6}, \\
& \mathcal{S}_{2}=\frac{1}{2} e-\frac{5}{12} e^{3}+\frac{1}{24} e^{5}, \\
& \mathcal{S}_{3}=\frac{3}{8} e^{2}-\frac{51}{128} e^{4}+\frac{543}{5120} e^{6}, \\
& \mathcal{S}_{4}=\frac{1}{3} e^{3}-\frac{13}{30} e^{5}, \\
& \mathcal{S}_{5}=\frac{125}{384} e^{4}-\frac{4625}{9216} e^{6}, \\
& \mathcal{S}_{6}=\frac{27}{80} e^{5}, \\
& \mathcal{S}_{7}=\frac{16807}{46080} e^{6}
\end{aligned}
$$


we refer the reader to [22] or any other standard book of celestial mechanics for the general expressions of the coefficients $\mathcal{C}_{k}(e)$ and $\mathcal{S}_{k}(e)$.

\section{APPENDIX B: THE APPROXIMATE METRIC COMPONENTS FOR CIRCULAR ORBITS}

We provide the expressions, to the relevant order in $A$ and $\Omega$, of the components of the metrics $\tilde{g}_{\alpha \beta}$ and $\tilde{\gamma}_{j k}$ [see Eqs. (3.8), (3.9), (3.10) and (3.12)], for a NS in circular orbit, using the phase model (4.12) in the two asymptotic limits $\Omega \gg 1$ and $\Omega \ll 1$.

\section{Asymptotic limit of several orbits}

We derive the expressions of the components of $\tilde{g}_{\alpha \beta}$ $(\alpha, \beta=0,1,2,3)$ and $\tilde{\gamma}_{j k}(i, j=1,2,3)$ given by Eq. (3.12) for $\Omega \gg 1$. We recall that, in general, $A \ll 1 / \Omega$. The time derivatives are given in Eq. (4.13), and the time averages are computed through Eq. (3.9).

To the leading order in $A$ and $\Omega$ the elements of $\tilde{g}_{\alpha \beta}$ read

$$
\begin{aligned}
& \tilde{g}_{00}=\frac{1}{12}, \\
& \tilde{g}_{01}=-\frac{\cos \alpha}{\Omega^{2}}+\frac{\cos (\alpha+\Omega)}{\Omega^{2}}, \\
& \tilde{g}_{02}=\frac{A \cos (\alpha+\Omega)}{2 \Omega}, \\
& \tilde{g}_{03}=\frac{A \cos \alpha}{2 \Omega}+\frac{A \cos (\alpha+\Omega)}{2 \Omega}, \\
& \tilde{g}_{11}=\frac{1}{2}-\frac{\sin (2 \alpha)}{4 \Omega}+\frac{\sin [2(a+\Omega)]}{4 \Omega}, \\
& \tilde{g}_{12}=\frac{A \cos [2(\alpha+\Omega)]}{4 \Omega}, \\
& \tilde{g}_{22}=\frac{A^{2}}{6}, \\
& \tilde{g}_{13}=-\frac{A \cos 2 \alpha}{2 \Omega}+\frac{A \cos 2(\alpha+\Omega)}{2 \Omega},
\end{aligned}
$$

Substituting these components into Eq. (3.10), which is related to $\tilde{\gamma}_{j k}$ through Eq. (3.12), one derives the following expressions, to the leading order in $A$ and $\Omega$ :

$$
\begin{aligned}
& \tilde{\gamma}_{11}=\frac{1}{2}-\frac{\sin (2 \alpha)}{4 \Omega}+\frac{\sin [2(a+\Omega)]}{4 \Omega}, \\
& \tilde{\gamma}_{12}=\frac{A \cos [2(\alpha+\Omega)]}{4 \Omega}, \\
& \tilde{\gamma}_{13}=-\frac{A \cos \alpha^{2}}{2 \Omega}+\frac{A \cos ^{2}(\alpha+\Omega)}{2 \Omega}, \\
& \tilde{\gamma}_{22}=\frac{A^{2}}{6}, \\
& \tilde{\gamma}_{23}=\frac{A^{2}}{4}, \\
& \tilde{\gamma}_{33}=\frac{A^{2}}{2} .
\end{aligned}
$$

The determinant (4.14) is obtained from the latter components by retaining the leading order terms in $A$ and $1 / \Omega$.

\section{Asymptotic limit of a fraction of one orbit}

The expression of the determinant in the case $\Omega \ll 1$ can be simply derived by Taylor expanding the components of $\tilde{g}_{\alpha \beta}$ and $\tilde{\gamma}_{j k}$ in powers of $\Omega$. Here we present the expressions of $\tilde{g}_{\alpha \beta}$ up to $\Omega^{10}$ :

$$
\begin{aligned}
& \tilde{g}_{00}=\frac{1}{12}, \\
& \tilde{g}_{01}=\frac{-\left(\Omega^{2} \cos \alpha\right)}{24}+\frac{\Omega^{4} \cos \alpha}{360}-\frac{\Omega^{6} \cos \alpha}{13440}+\frac{\Omega^{8} \cos \alpha}{907200} \\
& -\frac{\Omega^{10} \cos \alpha}{95800320}-\frac{\Omega \sin \alpha}{12}+\frac{\Omega^{3} \sin \alpha}{80}-\frac{\Omega^{5} \sin \alpha}{2016} \\
& +\frac{\Omega^{7} \sin \alpha}{103680}-\frac{\Omega^{9} \sin \alpha}{8870400}, \\
& \tilde{g}_{02}=\frac{-(A \Omega \cos \alpha)}{12}+\frac{A \Omega^{3} \cos \alpha}{90}-\frac{A \Omega^{5} \cos \alpha}{2240} \\
& +\frac{A \Omega^{7} \cos \alpha}{113400}-\frac{A \Omega^{9} \cos \alpha}{9580032}-\frac{A \sin \alpha}{12}+\frac{3 A \Omega^{2} \sin \alpha}{80} \\
& -\frac{5 A \Omega^{4} \sin \alpha}{2016}+\frac{7 A \Omega^{6} \sin \alpha}{103680}-\frac{A \Omega^{8} \sin \alpha}{985600} \\
& +\frac{11 A \Omega^{10} \sin \alpha}{1132185600}
\end{aligned}
$$




$$
\begin{aligned}
& \tilde{g}_{03}=\frac{-(A \Omega \cos \alpha)}{12}+\frac{A \Omega^{3} \cos \alpha}{80}-\frac{A \Omega^{5} \cos \alpha}{2016} \\
& +\frac{A \Omega^{7} \cos \alpha}{103680}-\frac{A \Omega^{9} \cos \alpha}{8870400}+\frac{A \Omega^{2} \sin \alpha}{24} \\
& -\frac{A \Omega^{4} \sin \alpha}{360}+\frac{A \Omega^{6} \sin \alpha}{13440}-\frac{A \Omega^{8} \sin \alpha}{907200} \\
& +\frac{A \Omega^{10} \sin \alpha}{95800320}, \\
& \tilde{g}_{11}=\Omega^{2}\left(\frac{1}{24}-\frac{\cos (2 \alpha)}{24}\right)+\Omega^{6}\left(\frac{1}{40320}-\frac{43 \cos (2 \alpha)}{13440}\right) \\
& +\Omega^{10}\left(\frac{1}{479001600}-\frac{4097 \cos (2 \alpha)}{479001600}\right) \\
& +\Omega^{8}\left(-\frac{1}{3628800}+\frac{769 \cos (2 \alpha)}{3628800}\right) \\
& +\Omega^{4}\left(-\frac{1}{720}+\frac{17 \cos (2 \alpha)}{720}\right)+\frac{\Omega^{3} \sin (2 \alpha)}{24} \\
& -\frac{7 \Omega^{5} \sin (2 \alpha)}{720}+\frac{107 \Omega^{7} \sin (2 \alpha)}{120960}-\frac{163 \Omega^{9} \sin (2 \alpha)}{3628800} \text {, } \\
& \tilde{g}_{12}=\Omega\left(\frac{A}{24}-\frac{A \cos (2 \alpha)}{24}\right)+\Omega^{5}\left(\frac{A}{13440}-\frac{43 A \cos (2 \alpha)}{4480}\right) \\
& +\Omega^{9}\left(\frac{A}{95800320}-\frac{4097 A \cos (2 \alpha)}{95800320}\right)+\Omega^{7}\left(\frac{-A}{907200}\right. \\
& \left.+\frac{769 A \cos (2 \alpha)}{907200}\right)+\Omega^{3}\left(\frac{-A}{360}+\frac{17 A \cos (2 \alpha)}{360}\right) \\
& +\frac{A \Omega^{2} \sin (2 \alpha)}{16}-\frac{7 A \Omega^{4} \sin (2 \alpha)}{288} \\
& +\frac{107 A \Omega^{6} \sin (2 \alpha)}{34560}-\frac{163 A \Omega^{8} \sin (2 \alpha)}{806400} \\
& +\frac{709 A \Omega^{10} \sin (2 \alpha)}{87091200} \text {, }
\end{aligned}
$$

$$
\begin{aligned}
\tilde{g}_{13}= & \frac{A \Omega^{3} \cos (2 \alpha)}{24}-\frac{7 A \Omega^{5} \cos (2 \alpha)}{720}+\frac{107 A \Omega^{7} \cos (2 \alpha)}{120960} \\
& -\frac{163 A \Omega^{9} \cos (2 \alpha)}{3628800}+\frac{A \Omega^{2} \sin (2 \alpha)}{24} \\
& -\frac{17 A \Omega^{4} \sin (2 \alpha)}{720}+\frac{43 A \Omega^{6} \sin (2 \alpha)}{13440} \\
& -\frac{769 A \Omega^{8} \sin (2 \alpha)}{3628800}+\frac{4097 A \Omega^{10} \sin (2 \alpha)}{479001600},
\end{aligned}
$$

$$
\begin{aligned}
\tilde{g}_{22}= & \frac{A^{2}}{24}-\frac{A^{2} \cos (2 \alpha)}{24}+\Omega^{4}\left(\frac{-A^{2}}{5760}-\frac{113 A^{2} \cos (2 \alpha)}{4480}\right) \\
& +\Omega^{8}\left(\frac{-A^{2}}{43545600}-\frac{93173 A^{2} \cos (2 \alpha)}{479001600}\right) \\
& +\Omega^{10}\left(\frac{A^{2}}{6706022400}+\frac{75547 A^{2} \cos (2 \alpha)}{9686476800}\right) \\
& +\Omega^{6}\left(\frac{A^{2}}{403200}+\frac{10999 A^{2} \cos (2 \alpha)}{3628800}\right) \\
& +\Omega^{2}\left(\frac{A^{2}}{144}+\frac{59 A^{2} \cos (2 \alpha)}{720}\right)+\frac{A^{2} \Omega \sin (2 \alpha)}{12} \\
& -\frac{19 A^{2} \Omega^{3} \sin (2 \alpha)}{360}+\frac{29 A^{2} \Omega^{5} \sin (2 \alpha)}{3024} \\
& -\frac{1489 A^{2} \Omega^{7} \sin (2 \alpha)}{1814400}+\frac{4913 A^{2} \Omega^{9} \sin (2 \alpha)}{119750400},
\end{aligned}
$$

$$
\begin{aligned}
\tilde{g}_{23}= & \Omega^{4}\left(\frac{-A^{2}}{1440}-\frac{7 A^{2} \cos (2 \alpha)}{288}\right)+\Omega^{8}\left(\frac{-A^{2}}{7257600}\right. \\
& \left.-\frac{163 A^{2} \cos (2 \alpha)}{806400}\right)+\Omega^{10}\left(\frac{A^{2}}{958003200}\right. \\
& \left.+\frac{709 A^{2} \cos (2 \alpha)}{87091200}\right)+\Omega^{6}\left(\frac{A^{2}}{80640}+\frac{107 A^{2} \cos (2 \alpha)}{34560}\right) \\
& +\Omega^{2}\left(\frac{A^{2}}{48}+\frac{A^{2} \cos (2 \alpha)}{16}\right)+\frac{A^{2} \Omega \sin (2 \alpha)}{24} \\
& -\frac{17 A^{2} \Omega^{3} \sin (2 \alpha)}{360}+\frac{43 A^{2} \Omega^{5} \sin (2 \alpha)}{4480} \\
& -\frac{769 A^{2} \Omega^{7} \sin (2 \alpha)}{907200}+\frac{4097 A^{2} \Omega^{9} \sin (2 \alpha)}{95800320},
\end{aligned}
$$

$$
\begin{aligned}
\tilde{g}_{33}= & \Omega^{4}\left(\frac{-A^{2}}{720}-\frac{17 A^{2} \cos (2 \alpha)}{720}\right)+\Omega^{8}\left(\frac{-A^{2}}{3628800}\right. \\
& \left.-\frac{769 A^{2} \cos (2 \alpha)}{3628800}\right)+\Omega^{6}\left(\frac{A^{2}}{40320}+\frac{43 A^{2} \cos (2 \alpha)}{13440}\right) \\
& +\Omega^{10}\left(\frac{A^{2}}{479001600}+\frac{4097 A^{2} \cos (2 \alpha)}{479001600}\right) \\
& +\Omega^{2}\left(\frac{A^{2}}{24}+\frac{A^{2} \cos (2 \alpha)}{24}\right)-\frac{A^{2} \Omega^{3} \sin (2 \alpha)}{24} \\
& +\frac{7 A^{2} \Omega^{5} \sin (2 \alpha)}{720}-\frac{107 A^{2} \Omega^{7} \sin (2 \alpha)}{120960} \\
& +\frac{163 A^{2} \Omega^{9} \sin (2 \alpha)}{3628800} .
\end{aligned}
$$

Substituting the former components into Eq. (3.10), which is related to $\tilde{\gamma}_{j k}$ through Eq. (3.12), one derives the following expressions for the components of the metric $\tilde{\gamma}_{j k}$ up to order $\Omega^{10}$ : 


$$
\begin{aligned}
& \tilde{\gamma}_{11}=\Omega^{6}\left(-\frac{1}{50400}-\frac{19 \cos (2 \alpha)}{50400}\right)+\Omega^{10}\left(-\frac{1}{419126400} \quad \tilde{\gamma}_{23}=\Omega^{4}\left(\frac{A^{2}}{2880}-\frac{A^{2} \cos (2 \alpha)}{576}\right)+\Omega^{8}\left(\frac{A^{2}}{7257600}\right.\right. \\
& \left.\left.-\frac{521 \cos (2 \alpha)}{209563200}\right)+\Omega^{8}\left(\frac{1}{3628800}+\frac{23 \cos (2 \alpha)}{518400}\right) \quad-\frac{41 A^{2} \cos (2 \alpha)}{806400}\right)+\Omega^{10}\left(\frac{-A^{2}}{838252800}\right. \\
& \left.+\Omega^{4}\left(\frac{1}{1440}+\frac{\cos (2 \alpha)}{1440}\right)-\frac{\Omega^{5} \sin (2 \alpha)}{1440} \quad+\frac{2027 A^{2} \cos (2 \alpha)}{762048000}\right)+\Omega^{6}\left(\frac{-A^{2}}{100800}+\frac{11 A^{2} \cos (2 \alpha)}{21600}\right) \\
& +\frac{11 \Omega^{7} \sin (2 \alpha)}{75600}-\frac{41 \Omega^{9} \sin (2 \alpha)}{3628800} \text {, } \\
& \tilde{\gamma}_{12}=\Omega^{5}\left(\frac{-A}{16800}-\frac{19 A \cos (2 \alpha)}{16800}\right)+\Omega^{9}\left(\frac{-A}{83825280}\right. \\
& \left.-\frac{521 A \cos (2 \alpha)}{41912640}\right)+\Omega^{7}\left(\frac{A}{907200}+\frac{23 A \cos (2 \alpha)}{129600}\right) \\
& +\Omega^{3}\left(\frac{A}{720}+\frac{A \cos (2 \alpha)}{720}\right)-\frac{A \Omega^{4} \sin (2 \alpha)}{576} \\
& +\frac{11 A \Omega^{6} \sin (2 \alpha)}{21600}-\frac{41 A \Omega^{8} \sin (2 \alpha)}{806400} \\
& +\frac{2027 A \Omega^{10} \sin (2 \alpha)}{762048000}, \\
& \tilde{\gamma}_{13}=\frac{-\left(A \Omega^{5} \cos (2 \alpha)\right)}{1440}+\frac{11 A \Omega^{7} \cos (2 \alpha)}{75600} \\
& \tilde{\gamma}_{33}=\Omega^{4}\left(\frac{A^{2}}{1440}-\frac{A^{2} \cos (2 \alpha)}{1440}\right)+\Omega^{8}\left(\frac{A^{2}}{3628800}\right. \\
& \left.-\frac{23 A^{2} \cos (2 \alpha)}{518400}\right)+\Omega^{10}\left(\frac{-A^{2}}{419126400}\right. \\
& \left.+\frac{521 A^{2} \cos (2 \alpha)}{209563200}\right)+\Omega^{6}\left(\frac{-A^{2}}{50400}+\frac{19 A^{2} \cos (2 \alpha)}{50400}\right) \\
& +\frac{A^{2} \Omega^{5} \sin (2 \alpha)}{1440}-\frac{11 A^{2} \Omega^{7} \sin (2 \alpha)}{75600} \\
& +\frac{41 A^{2} \Omega^{9} \sin (2 \alpha)}{3628800} \text {. }
\end{aligned}
$$$$
-\frac{41 A \Omega^{9} \cos (2 \alpha)}{3628800}-\frac{A \Omega^{4} \sin (2 \alpha)}{1440}
$$$$
+\frac{19 A \Omega^{6} \sin (2 \alpha)}{50400}-\frac{23 A \Omega^{8} \sin (2 \alpha)}{518400}
$$$$
+\frac{521 A \Omega^{10} \sin (2 \alpha)}{209563200} \text {, }
$$$$
\tilde{\gamma}_{22}=\Omega^{4}\left(\frac{A^{2}}{50400}-\frac{23 A^{2} \cos (2 \alpha)}{7200}\right)+\Omega^{8}\left(\frac{13 A^{2}}{838252800}\right.
$$$$
\left.-\frac{48509 A^{2} \cos (2 \alpha)}{838252800}\right)+\Omega^{10}\left(\frac{-A^{2}}{7925299200}\right.
$$$$
\left.+\frac{413747 A^{2} \cos (2 \alpha)}{145297152000}\right)+\Omega^{6}\left(\frac{-A^{2}}{907200}\right.
$$$$
\left.+\frac{599 A^{2} \cos (2 \alpha)}{907200}\right)+\Omega^{2}\left(\frac{A^{2}}{360}+\frac{A^{2} \cos (2 \alpha)}{360}\right)
$$$$
-\frac{A^{2} \Omega^{3} \sin (2 \alpha)}{240}+\frac{503 A^{2} \Omega^{5} \sin (2 \alpha)}{302400}
$$$$
-\frac{193 A^{2} \Omega^{7} \sin (2 \alpha)}{907200}+\frac{114431 A^{2} \Omega^{9} \sin (2 \alpha)}{8382528000},
$$

The determinant (4.23) is then derived from the latter components and Taylor expanding the resulting expression to the leading order in $\Omega$.

\section{APPENDIX C: THE APPROXIMATE METRIC COMPONENTS FOR ELLIPTIC ORBITS}

Below we list the metric components $\tilde{g}_{\alpha \beta}$ for $\alpha \geqslant \beta$, in the coordinates $\kappa, X, Y, e, \psi, \alpha$ up to order $e^{2}$ and in the leading order $X, Y$ for the case $\Omega \gg 1$ :

$$
\begin{aligned}
& \tilde{g}_{00}=\frac{1}{12}, \\
& \tilde{g}_{0 X}=-\frac{1}{4} e^{2} X, \\
& \tilde{g}_{0 Y}=0, \\
& \tilde{g}_{0 e}=-\frac{1}{4} e X^{2}, \\
& \tilde{g}_{0 \Omega}=0, \\
& \tilde{g}_{0 \alpha}=0, \\
& \tilde{g}_{X X}=\frac{1}{2}\left(1-\frac{1}{2} e^{2}\right),
\end{aligned}
$$




$$
\begin{aligned}
& \tilde{g}_{X Y}=0, \\
& \tilde{g}_{X e}=-\frac{1}{4} e X, \\
& \tilde{g}_{X \Omega}=\frac{1}{4}\left(1-\frac{1}{2} e^{2}\right) Y, \\
& \tilde{g}_{X \alpha}=\frac{1}{2}\left(1-\frac{1}{2} e^{2}\right) Y, \\
& \tilde{g}_{Y Y}=\frac{1}{2}\left(1-e^{2}\right), \\
& \tilde{g}_{Y e}=-\frac{1}{2} e Y, \\
& \tilde{g}_{Y \Omega}=-\frac{1}{4}\left(1-\frac{1}{2} e^{2}\right) X, \\
& \tilde{g}_{Y \alpha}=-\frac{1}{2}\left(1-\frac{1}{2} e^{2}\right) X, \\
& \tilde{g}_{e e}=\frac{1}{8}\left(X^{2}+Y^{2}\right)+\frac{e^{2}}{16}\left(X^{2}+7 Y^{2}\right),
\end{aligned}
$$

$$
\begin{aligned}
& \tilde{g}_{e \Omega}=\frac{1}{8} e X Y, \\
& \tilde{g}_{e \alpha}=\frac{1}{4} e X Y, \\
& \tilde{g}_{\Omega \Omega}=\frac{1}{6}\left(X^{2}+Y^{2}\right)+\frac{e^{2}}{24}\left(X^{2}-Y^{2}\right), \\
& \tilde{g}_{\Omega \alpha}=\frac{1}{4}\left(X^{2}+Y^{2}\right)+\frac{e^{2}}{16}\left(X^{2}-Y^{2}\right), \\
& \tilde{g}_{\alpha \alpha}=\frac{1}{2}\left(X^{2}+Y^{2}\right)+\frac{e^{2}}{8}\left(X^{2}-Y^{2}\right) .
\end{aligned}
$$

In the previous expressions we have also dropped all the terms of order $\Omega^{-1}$ and higher, and set $\alpha=0$. Substituting these components $\tilde{g}_{j k}$ into Eq. (3.10), and consistently working within the appropriate approximations, one can derive (to the leading order in $e^{2}$ ) the expression for the determinant in Eq. (4.28).
[1] A. Abramovici et al., Science 256, 325 (1992).

[2] C. Bradaschia et al., Nucl. Instrum. Methods Phys. Res. A 289, 518 (1990).

[3] K. Danzmann, in Gravitational Wave Experiments, edited by E. Coccia, G. Pizzella and F. Ronga (World Scientific, Singapore, 1995), pp. 100-111.

[4] K. Tsubono, in Gravitational Wave Experiments, edited by E. Coccia, G. Pizzella and F. Ronga (World Scientific, Singapore, 1995), pp. 112-114.

[5] R. J. Sandeman et al., A.I.G.O. Prospectus, 1997.

[6] M. Cerdonio, in Gravitation and Relativity: At the turn of the millennium, edited by N. Dadhich and J. Narlikar (IUCAA, Pune, 1998), pp. 211-230.

[7] P. L. Bender et al., LISA Pre-Phase A Report, 2nd ed., Report No. MPQ 233, 1995.

[8] B. Bertotti, A. Vecchio, and L. Iess, Phys. Rev. D 59, 082001 (1999).

[9] K. S. Thorne, in 300 Years of Gravitational, edited by S. W. Hawking and W. Israel (Cambridge University Press, Cambridge, England, 1987), pp. 330-458.

[10] K. S. Thorne, in Proceedings of the Snowmass 95 Summer Study on Particle and Nuclear Astrophysics and Cosmology, edited by E. W. Kolb and R. Peccei (World Scientific, Singapore, 1995), p. 398.

[11] E. Flanagan, in Gravitation and Relativity: At the Turn of the Millennium [6], pp. 177-198.

[12] B. F. Schutz, Class. Quantum Grav. 16, A131 (1999).

[13] B. F. Schutz, in The Detection of Gravitational Waves, edited by D. Blair (Cambridge University Press, Cambridge, England, 1989), pp. 406-427.

[14] P. R. Brady, T. Creighton, C. Cutler, and B. F. Schutz, Phys. Rev. D 57, 2101 (1998).

[15] P. R. Brady and T. Creighton, Phys. Rev. D 61, 082001 (2000).

[16] B. F. Schutz and M. A. Papa, in Gravitational Waves and Experimental Gravity, Proceedings of Moriond, 1999 (Editions Frontieres, Orsay, 1999).

[17] J. H. Taylor, R. N. Manchester, and A. G. Lyne, Astrophys. J., Suppl. Ser. 88, 529 (1993); J. H. Taylor, R. N. Manchester, A. G. Lyne and F. Camillo (unpublished). The version of the catalog used in this paper is actually the electronic, up-dated version of the original one. It is the Catalogue of 706 pulsars, available via a anonymous ftp at pulsar.princeton.edu. Note that after the publication of the catalogue of 706 pulsars more binary systems have been found.

[18] J. Papaloizou and J. E. Pringle, Mon. Not. R. Astron. Soc. 184, 501 (1978).

[19] R.V. Wagoner, Astrophys. J. 278, 345 (1984).

[20] L. Bildsten, Astrophys. J. Lett. 501, L89 (1998).

[21] G. Ushomirsky, C. Cutler, and L. Bildsten, Mon. Not. R. Astron. Soc. 319, 902 (2000).

[22] L. G. Taff, Celestial Mechanics (Wiley, New York, 1985), pp. $58-61$.

[23] S. V. Dhurandhar and B. S. Sathyaprakash, Phys. Rev. D 49, 1707 (1994).

[24] R. Balasubramanian, B. S. Sathyaprakash, and S. V. 
Dhurandhar, Phys. Rev. D 53, 3033 (1996).

[25] B. J. Owen, Phys. Rev. D 53, 6749 (1996).

[26] B. F. Schutz, "Source of radiation from neutron stars,' available at http://www.lsc-group.phys.uwm.edu/lscasis/

[27] C. Cutler and D. I. Jones, Phys. Rev. D 63, 024002 (2001).

[28] S. B. Anderson, P. M. Gorham, S. R. Kulkarni, T. A. Prince, and A. Wolszczan, Nature (London) 346, 42 (1990).

[29] R. N. Manchester et al., Mon. Not. R. Astron. Soc. 279, 1235 (1996).

[30] A. G. Lyne et al., Mon. Not. R. Astron. Soc. 295, 743 (1998).

[31] F. Camilo et al., "The Parkes multi-beam pulsar survey,", astro-ph/9911185.
[32] The main parameters of the newly discovered pulsars with the survey at Parkes can be found at the following URL: http:// www.atnf.csiro.au/ pulsar/psr/pmsurv/pmwww/pmpsrs.db

[33] E. W. Gottlieb, E. L. Wright, and W. Liller, Astrophys. J., Lett. Ed. 195, L33 (1975).

[34] A. P. Cowley and D. Crampton, Astrophys. J., Lett. Ed. 201, L65 (1975).

[35] H. M. Johnston and S. R. Kulkarni, Astrophys. J. 368, 504 (1991).

[36] S. M. Ransom, "A fast search technique for binary pulsars," astro-ph/9911073. 